\title{
EchoGéo
}

$11 \mid 2010$

Madagascar

\section{Le film du temps en Imerina (Madagascar) : dialogue entre lecture paysanne et lecture «scientifique »}

Daniel Peyrusaubes

\section{CpenEdition}

Journals

Édition électronique

URL : https://journals.openedition.org/echogeo/11667

DOI : 10.4000/echogeo. 11667

ISSN : 1963-1197

Éditeur

Pôle de recherche pour l'organisation et la diffusion de l'information géographique (CNRS UMR 8586)

Référence électronique

Daniel Peyrusaubes, « Le film du temps en Imerina (Madagascar) : dialogue entre lecture paysanne et lecture « scientifique » », EchoGéo [En ligne], 11 | 2010, mis en ligne le 24 février 2010, consulté le 01 août 2021. URL : http://journals.openedition.org/echogeo/11667 ; DOI : https://doi.org/10.4000/ echogeo. 11667

Ce document a été généré automatiquement le 1 août 2021.

EchoGéo est mis à disposition selon les termes de la licence Creative Commons Attribution - Pas d'Utilisation Commerciale - Pas de Modification 4.0 International (CC BY-NC-ND) 


\title{
Le film du temps en Imerina (Madagascar) : dialogue entre lecture paysanne et lecture « scientifique »
}

\author{
Daniel Peyrusaubes
}

1 Dans le contexte du débat scientifique mondial sur le changement global, l'objet de ce texte est de présenter et de confronter deux appréhensions du climat d'une partie des hautes terres centrales de l'Imerina, à Madagascar. La première approche est d'origine paysanne, à partir d'entretiens réalisés dans la campagne malgache; la seconde relève d'une lecture physique du fonctionnement atmosphérique de cette région de l'ile Rouge.

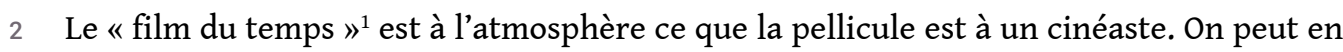
effet faire un parallèle entre un film qui est une suite de plans organisés avec précision, et un climat qui se décompose à son tour non pas en plans mais en séquences (de temps).

3 L'angle d'attaque choisi de cette double lecture du climat relève du concept universel de saison, permettant aux hommes de se repérer dans le temps qui passe à travers le temps qu'il fait. Une caractéristique majeure de ce concept est la vision quadripartite du climat avec l'ordre immuable du printemps, de l'été, de l'automne et de l'hiver. Dans le domaine atmosphérique qui nous intéresse, on trouve également cette division en quatre au travers des qualités élémentaires de l'enveloppe gazeuse terrestre : chaud, froid, sec, humide.

4 Aussi, l'analyse d'un climat ne peut échapper, dans un premier temps tout au moins, à cette tendance lourde de la vision quaternaire des choses. Est-ce pour autant qu'il existe systématiquement quatre saisons où que l'on se trouve sur la planète? La réponse est non car les climats de la Terre sont très divers, conséquence d'un système climatique fort complexe. Quid alors de cette posture culturelle, et des perceptions 
humaines confrontées à la quotidienneté ? L'analyse du climatologue de la chronologie du temps qu'il fait à l'échelle annuelle est-elle en phase avec celle du terrien lambda?

5 Nous nous sommes posés cette question dans le cadre d'une recherche en géoclimatologie effectuée sur les Hautes Terres de Madagascar, précisément en Imerina centre-oriental ${ }^{2}$. Parmi les Etats les plus ruraux du monde, ce pays se caractérise par une population paysanne nombreuse, et particulièrement sensible aux «choses du temps qu'il fait». Dès lors, à quel découpage temporel cette sensibilité climatique aboutit-elle ? Fait-il consensus au sein de la communauté paysanne ? Est-ce un élément important dans la culture merina ? Parallèlement, la lecture du climatologue est-elle en cohérence ou non avec celle de l'agriculteur malgache? L'analyse en surface et en altitude de paramètres atmosphériques spécifiques aboutit-elle à des convergences et/ ou des contradictions par rapport aux savoirs et perçus locaux? Au-delà des résultats et des limites de la comparaison, ces deux approches très dissemblables du déroulement des saisons participent finalement de l'approfondissement des connaissances tant de l'environnement climatique que du fonctionnement de la société rurale de cette région de Madagascar. En fait, nous cherchons à formaliser un dialogue encore trop caché entre une population et son environnement climatique, ceci sous le regard du scientifique.

6 Une présentation générale de l'espace géographique concerné précède notre attention portée sur le découpage saisonnier paysan ressortant d'entretiens réalisés dans la campagne merina. Est exhumée ici une véritable météoroculture populaire. La lecture du géoclimatologue est ensuite exposée, s'appuyant sur une étude analytique de quelques descripteurs physiques de la troposphère des Hautes Terres centrales malgaches. Un essai de confrontation des calendriers est proposé.

\section{L'Imerina centre-oriental, des Hautes Terres à la forêt primaire de l'Est}


Figure 1 - Madagascar, au sud-ouest de l'océan Indien

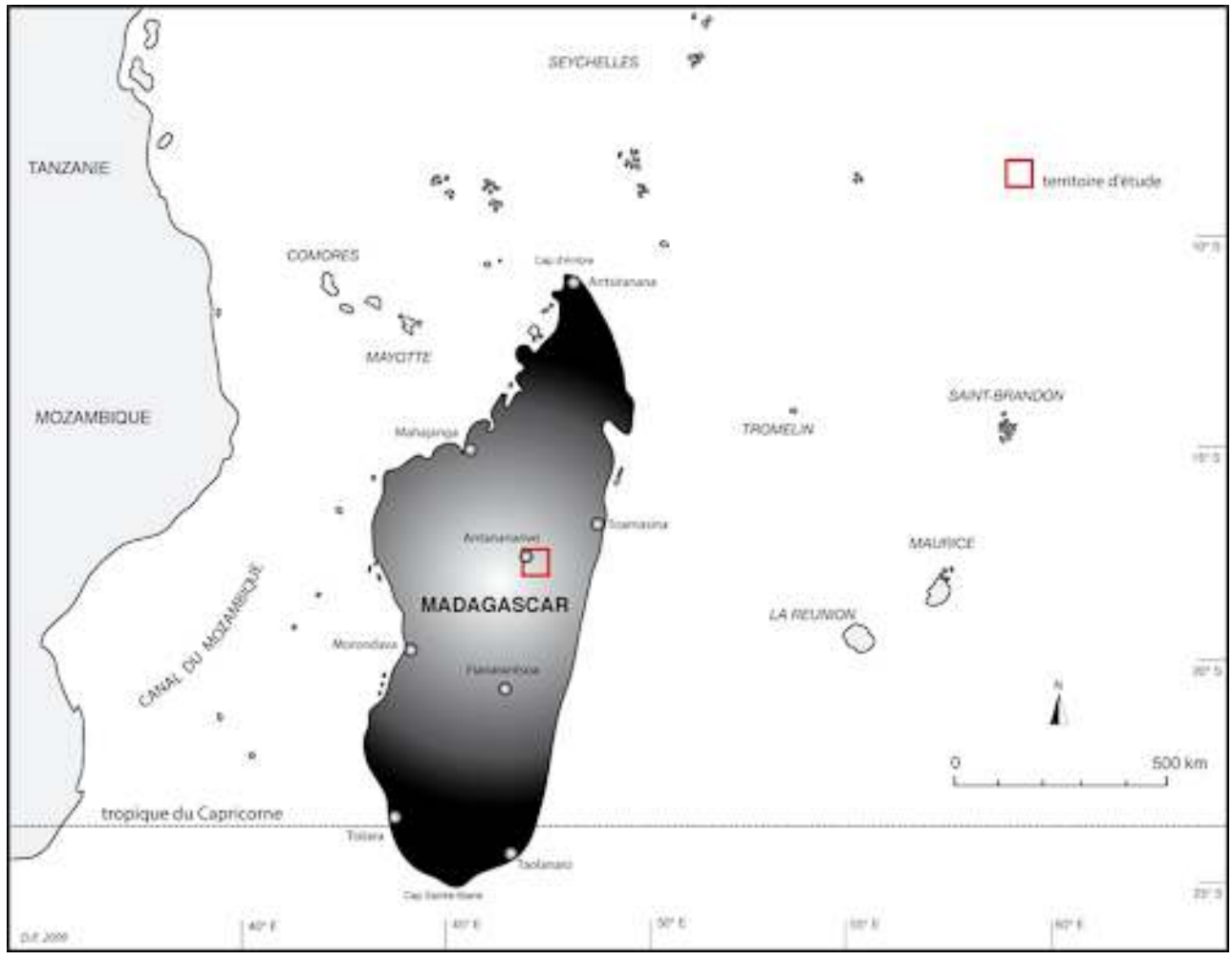

Réalisation : Daniel Peyrusaubes

7 Situé dans l'hémisphère sud, au sud-ouest du bassin océanique indien, l'île de Madagascar compte parmi les plus vastes espaces insulaires de la planète (fig.1).

8 L'Imerina se situe sur les hautes terres centrales du socle cristallin, entre $18^{\circ}$ et $20^{\circ}$ environ de latitude sud, et de part et d'autre de $47^{\circ}$ de longitude est. L'altitude de cet espace oscille entre 800 mètres en ses confins occidentaux et 1700 mètres dans sa partie la plus orientale. Les quelques sommets existants ne dépassent pas 1800 mètres, exception faite du massif volcanique de l'Ankaratra (2642 mètres au pic Tsiafajavona), à une cinquantaine de kilomètres au sud d'Antananarivo. Le facteur altitude est, par conséquent, une composante majeure de la géographie du pays merina ${ }^{3}$. Dans sa partie la plus orientale, la Falaise de l'Angavo dessine la ligne de partage des eaux entre façade est (océan Indien) et façade ouest (canal du Mozambique), et annonce l'amorce d'un gradient bioclimatique est-ouest très progressif. L'espace géographique de cette recherche (la moitié est de la figure 2) s'étend d'Antananarivo aux environs du lacréservoir de Tsiazompaniry, bien visible en sombre au sud-est sur la figure 2 . 
Figure.2 - L'Imerina : Antananarivo (centre-nord, violet clair), lacs de Mantasoa (nord-est) et de Tsiazompaniry (sud-est) distants entre eux d'environ $25 \mathrm{~km}$, forêt primaire (est, vert intense), terres cultivées (ouest, rosé)

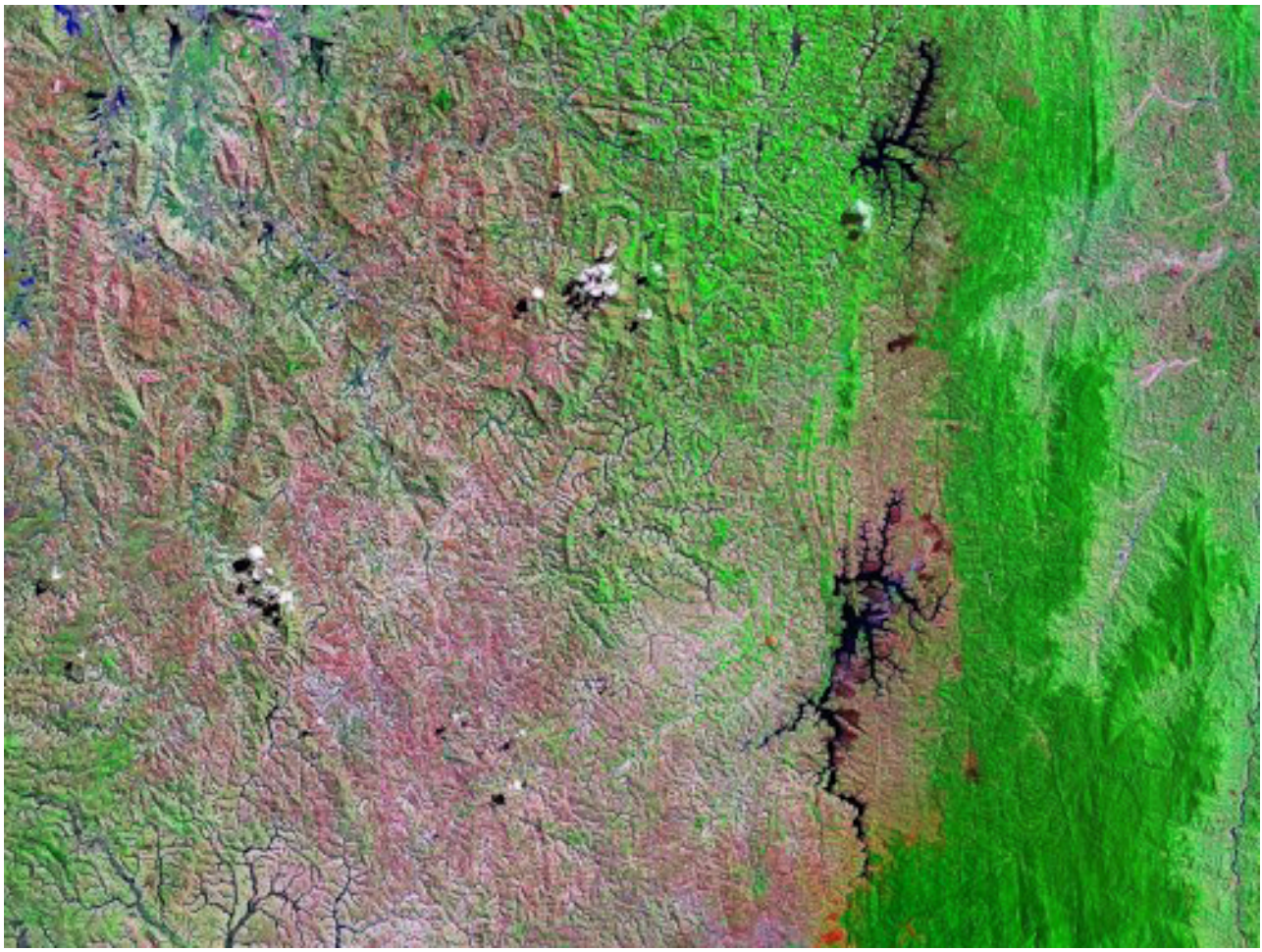

Source : https://zulu.ssc.nasa.gov/mrsid/mrsid.pl

9 Interfaces les plus directes et immédiates avec l'observateur, les paysages, où société et milieu sont en perpétuelle interaction, alternent collines d'allure émoussée et vallées pas très larges à fond plat. Les premières, les tanety, sont, sur leurs parties sommitales, soit dépouillées (roches granitiques à nu), soit coiffées de reboisements d'eucalyptus, parfois de mimosas ou de pins (fig.3). Elles portent sur leurs flancs convexo-concaves des cultures de contre-saison quand la pente n'est pas très forte, mais sont également aménagées en terrasses dans les secteurs les plus densément occupés. 
Figure 3 - Les immensités paysagères de l'Imerina oriental, entre collines cristallines émoussées et réseaux de vallées peu encaissées

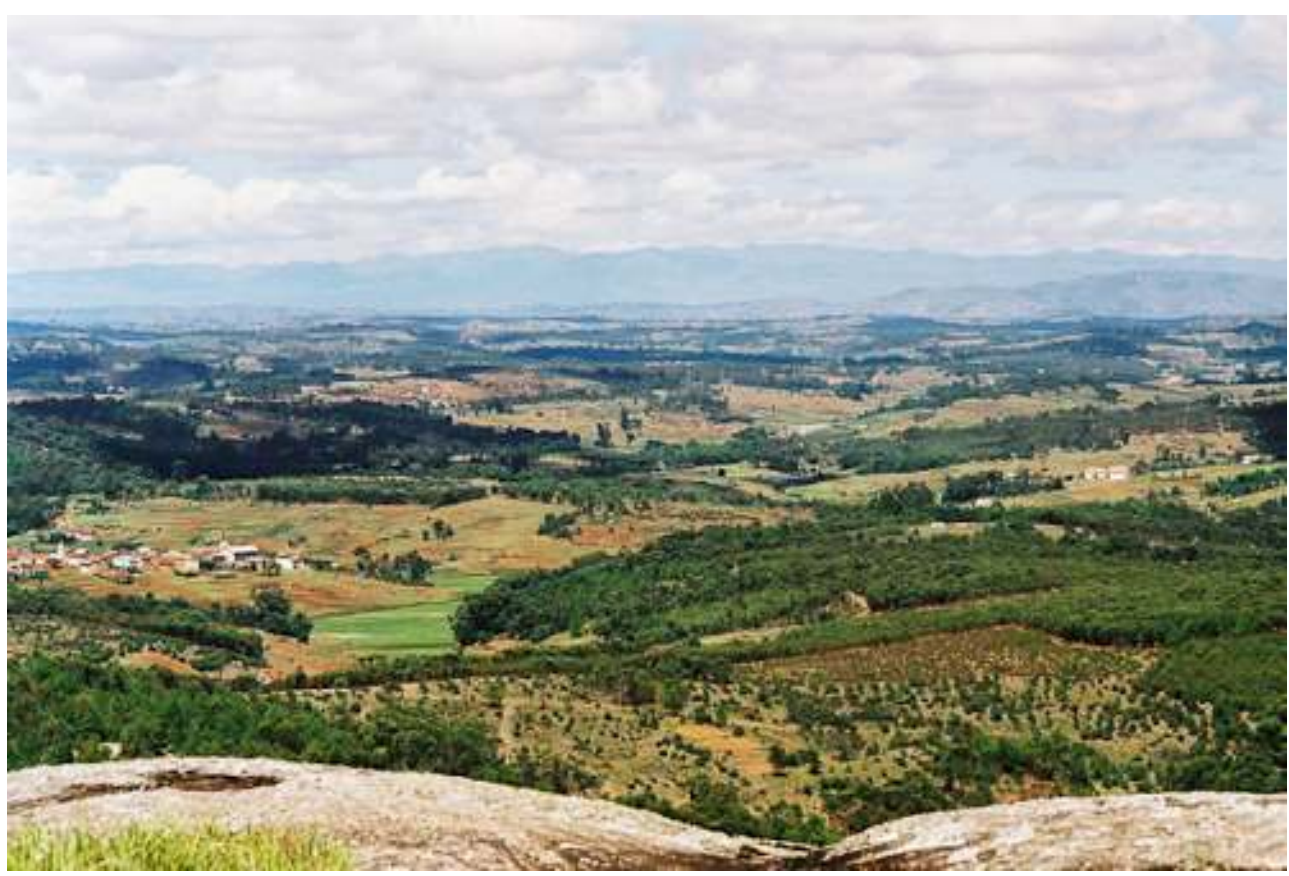

Source : Daniel Peyrusaubes

Les vallées sont, quant à elles, systématiquement cultivées en riz durant la saison pluvieuse, occupées par des plantes de contre-saison ${ }^{4}$ le reste de l'année. Aussi, l'habitat se situe de préférence sur les premières pentes des tanety. En hameaux ou isolées, les maisons rurales traditionnelles merina s'égrènent le long des vallées cultivées et reflètent, par leur implantation, un espace organisé (fig. 4). Au cours des saisons, le «film du temps " s'inscrit dans des constructions paysagères se parant de tonalités culturales très colorées, le tout sur le fond ocre des terres latéritiques. 
Figure 4 - Des vallées systématiquement cultivées en saison des pluies, des versants en cultures et/ou reboisés

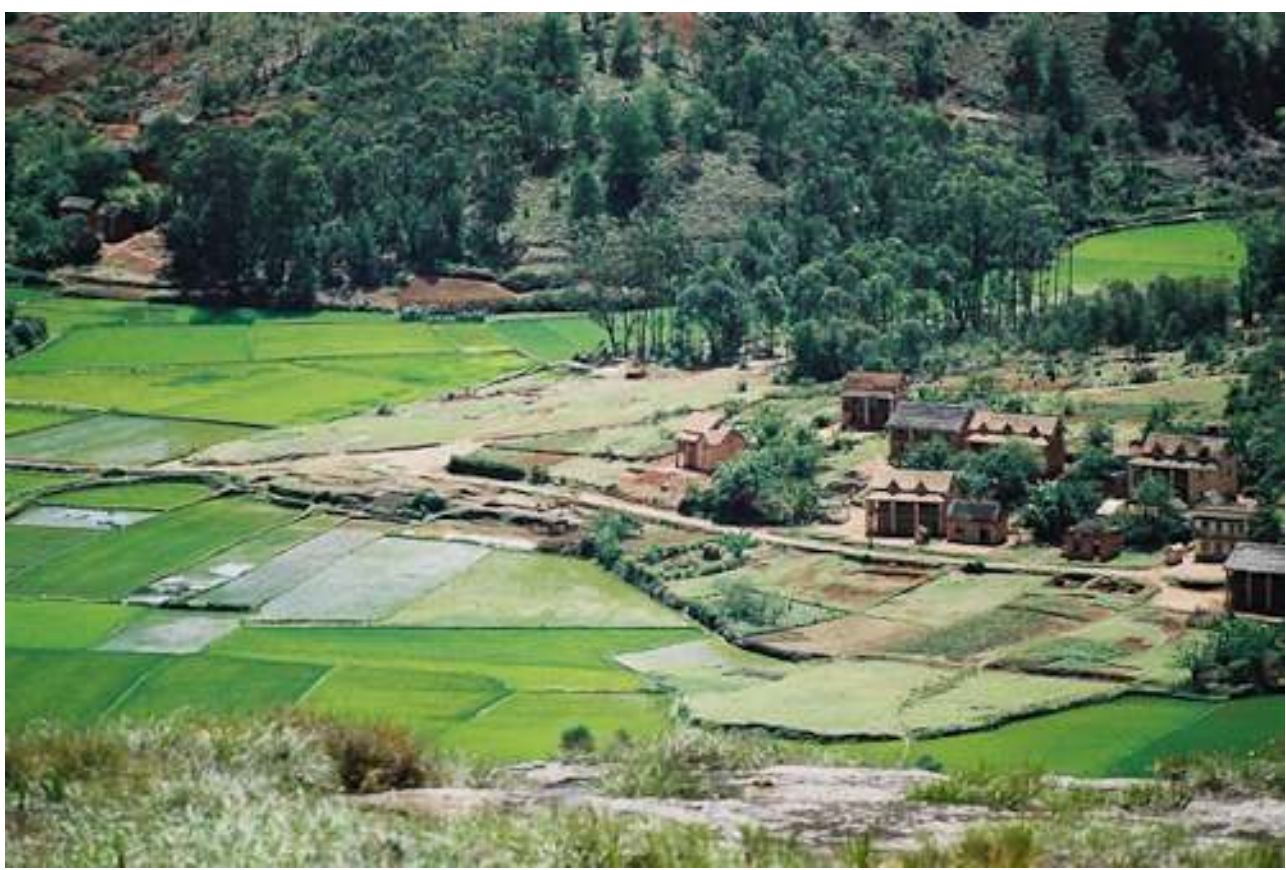

Source : Daniel Peurusaubes

\section{Le calendrier agro-climatique paysan, une scansion temporelle au cœur de la culture merina.}

\section{Une population paysanne pauvre, en contact permanent avec les éléments atmosphériques}

11 L'identification des saisons s'appuie sur les principaux résultats d'entretiens ${ }^{5}$ réalisés auprès de la communauté rurale de la région du lac de Tsiazompaniry, mais également à l'aide de quelques références littéraires. La grande sensibilité des populations agraires au climat régional est particulièrement aiguë dans les campagnes où l'artificialisation du milieu est faible, et où les rapports qu'entretient le paysan avec son environnement atmosphérique sont directs et quotidiens. L'individu vit dans l'intimité du climat, c'est le cas de figure qui s'observe fréquemment dans les pays en développement. Pour les populations interrogées, parler du climat n'a jamais suscité de réaction négative, bien au contraire. Quel que soit le lieu d'interview, nos interlocuteurs ont toujours répondu aux questions, certains y mettant même beaucoup d'énergie dans le ton. Leur aisance à parler de leur environnement climatique, situation d'ailleurs inédite pour tous ${ }^{6}$, confirme, a posteriori, cette idée de la prééminence du temps (qu'il fait) dans leur vie quotidienne. Ceci s'est particulièrement vérifié chez les paysans à temps complet, mais aussi chez les personnes exerçant une ou plusieurs activités (fonctionnaires, artisans,...). C'est l'exemple de ces femmes qui, à chaque fin septembre/début octobre, attendent le bon moment pour repiquer les jeunes plants de riz (fig.5). Ou celui de cet apiculteur qui vit les saisons avec la migration annuelle des abeilles (fig.6). C'est enfin le cas de cette famille paysanne rencontrée aux confins 
orientaux de l'Imerina, qui décrit avec justesse le gradient bioclimatique entre l'est et l'ouest des Hautes Terres centrales (fig.7).

Figure 5 - Le travail continuel de la terre, repiquage saisonnier du riz par les femmes

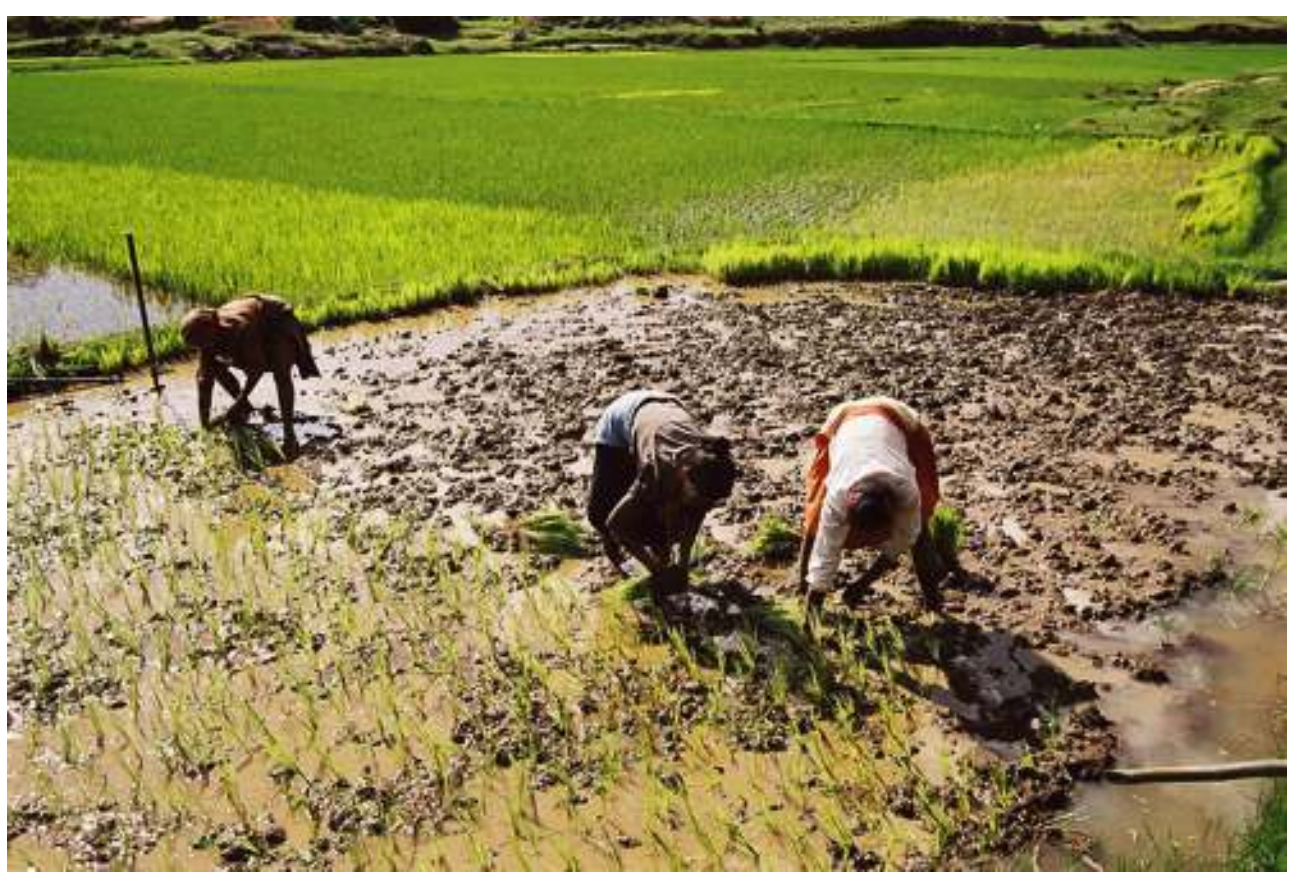

Source : Daniel Peyrusaubes

Figure 6 : L'apiculteur et sa ruche, observateur averti du temps qu'il fait

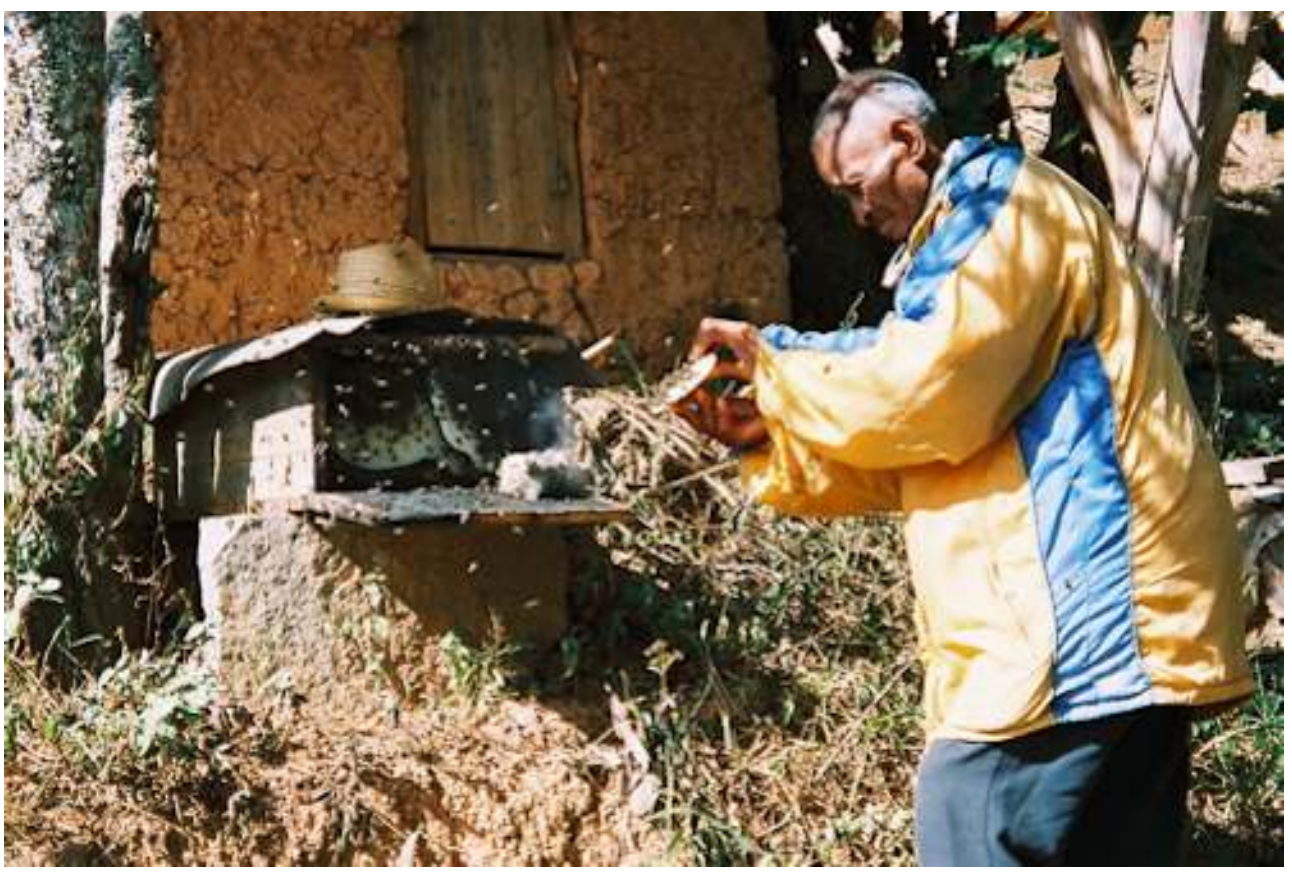

Source : Daniel Peyrusaubes 
Figure 7 - Famille paysanne pionnière de l'orient merina, aux avant-postes de l' « entre-deux-vents »

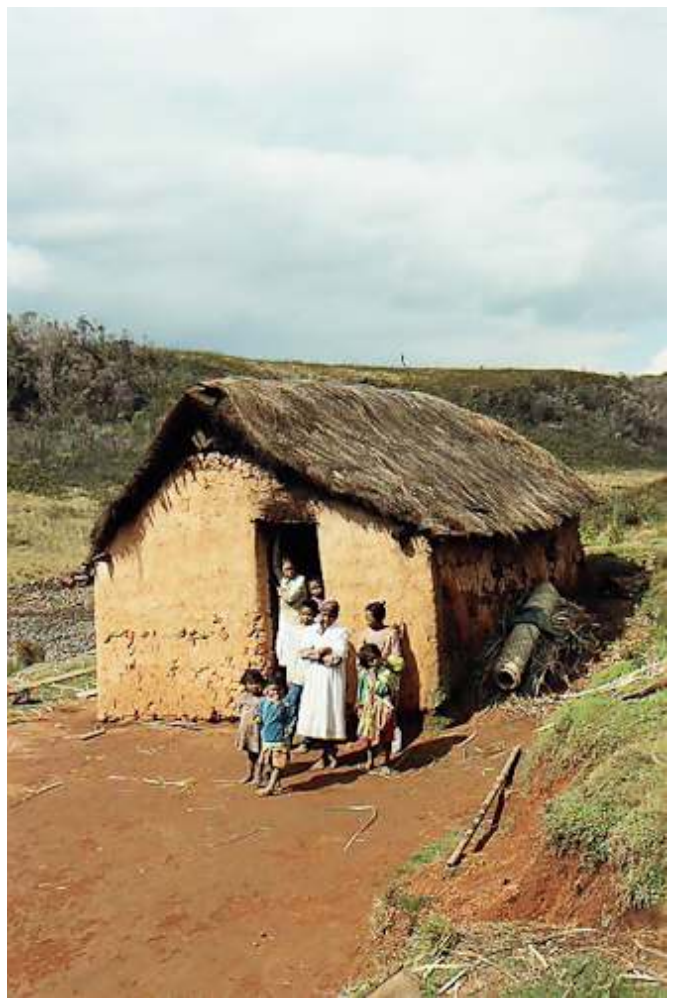

Source : Daniel Peyrusaubes

\section{Le découpage climatique paysan}

Ce thème a déjà fait l'objet de recherches approfondies par les spécialistes du milieu rural des Hautes Terres (Blanc-Pamard 1986, Rakoto Ramiarantsoa 1995). D'après ces recherches, trois ou quatre saisons sont généralement citées par les ruraux. Ainsi, l'exemple des communautés rurales de Mahitsy, au nord-ouest d'Antananarivo, soit à quelque $80 \mathrm{~km}$ au nord-ouest de notre terrain d'étude. Là, « ... les paysans apprennent au voyageur que l'année se divise en quatre saisons qui sont le fahavaratra ou 'moment du tonnerre' et qui correspond à la période agricole pluvieuse (fin octobre à mi-mars), le fararano ou 'dernière eau' et pendant lequel on récolte le riz (mars à mi-juin), le ririnina que caractérisent le froid et le vent (mi-juin à septembre), et le lohataona ou 'tête de l'année' et qui est marqué par la préparation des futurs champs (septembre à octobre) » (Blanc-Pamard, 1986). On le voit, l'énoncé des saisons est intimement lié à la culture du riz; on peut même affirmer qu'en Imerina le calendrier climatique se confond avec le calendrier rizicole, mais aussi avec celui des cultures de contre saison. De plus, du fait de la mosaïque climatique de l'île et de la multiplicité des groupes ethniques, l'appellation et la durée de ces saisons varient (Hébert, 1968).

Le nombre des saisons peut différer selon les lieux et les individus. Par exemple, chez les populations de l'Imerina oriental, «... Moment de la récolte, le fararano constitue un temps fort et marquant dans l'année, sans pour autant être individualisé comme une saison par les paysans. Il clôture le fahavaratra, sur le plan climatique et sur celui de l'alimentation, mais il n'en est pas exclu 臨...臨. Le terme fararano est d'ailleurs utilisé pour désigner l'époque («nous sommes en fararano ») comme l'acte («nous allons faire 
le fararano ») » (Rakoto Ramiarantsoa, 1995). Nos entretiens confirment ce fait puisque le terme lui-même n'a quasiment pas été cité.

D'une manière générale, la notion de saison est source d'avis divers auprès des individus. Certains (peu) comptent deux saisons, mais sans systématiquement les formuler de la même façon, en particulier pour leur période d'occurrence. Il en va ainsi d'une saison des pluies débutant en août (!) pour l'un, en octobre ou en novembre pour l'autre, mais s'achevant en mars presque pour tous; la saison «sèche" parcourt le reste de l'année ${ }^{7}$. Les termes de fahavaratra (la racine en est varatra, « orage ») et ririnina - la racine en désigne la froidure - sont d'abord cités pour spécifier les deux époques pluvieuse et peu humide- puis, dans un second temps, pour leur caractère thermique (mais seulement en "pays vieux ", c'est-à-dire en Imerina central). En ambavala, terres les plus à l'est de l'Imerina, les différences thermiques sont plus appuyées dans les récits, particulièrement pour ririnina; ici, la perception climatique est nettement différentielle.

Au-delà de ce découpage binaire, les plus sensibles des individus au phénomène des saisons en définissent alors trois, voire quatre, faisant alors intervenir principalement le printemps (lohataona) et, accessoirement, l'automne. A chacune de ces périodes sont alors associées une ou des caractéristiques ambiancielles. En été, le phénomène orageux, avec ou sans grêle, et d'importantes précipitations. En ririnina, de la bruine, du crachin (erika) et du froid, avec parfois des brouillards matinaux et encore quelques averses orageuses. Et un printemps habituellement décrit par des journées chaudes sans précipitation. Parfois est signalé le cas particulier du mois d'août, qualifié de mois intermédiaire entre ririnina et lohataona, car des types de temps contrastés peuvent s'y dérouler : alternance de journées froides à erika et de journées plus chaudes. Il y a, dans ces propos, un lien très fort entre le temps qui passe et le temps qu'il fait, en fait une sorte de «... socialisation de la durée... » comme l'évoque de La Soudière (1999).

\section{Observations paysannes de signaux saisonniers}

16 Décrire, évoquer, citer les saisons pour les Merina de la région de Tsiazompaniry, c'est aussi raconter des paysages changeants où les rizières, en saison des pluies, habillent de mille nuances de verts le moindre vallon.

Mais c'est également distiller de nouveaux signes, non atmosphériques ceux-là, et donnant plein sens au concept de saison. C'est fréquemment citer des oiseaux «... qui présentent une affinité toute particulière avec la météorologie. L'air et le ciel sont en effet leur territoire... » (De la Soudière, 1999). A l'image de ce petit volatile qui, de gris en ririnina, se pare d'un rouge éclatant en fahavaratra, et que l'on nomme fody (Foudia madagascariensis). Mais qui est chassé des rizières par les enfants, car trop gourmand en épis de riz mûrs!

18 A l'image de la fleur de sahondra (Aloe capitala) qui, sur les rochers granitiques et siliceux de la région, s'épanouit de juin à septembre, et donne ainsi une marque colorée à ririnina (fig.8). 
Figure 8 - Fleur de sahondra (Aloe capitala), marque colorée de ririnina

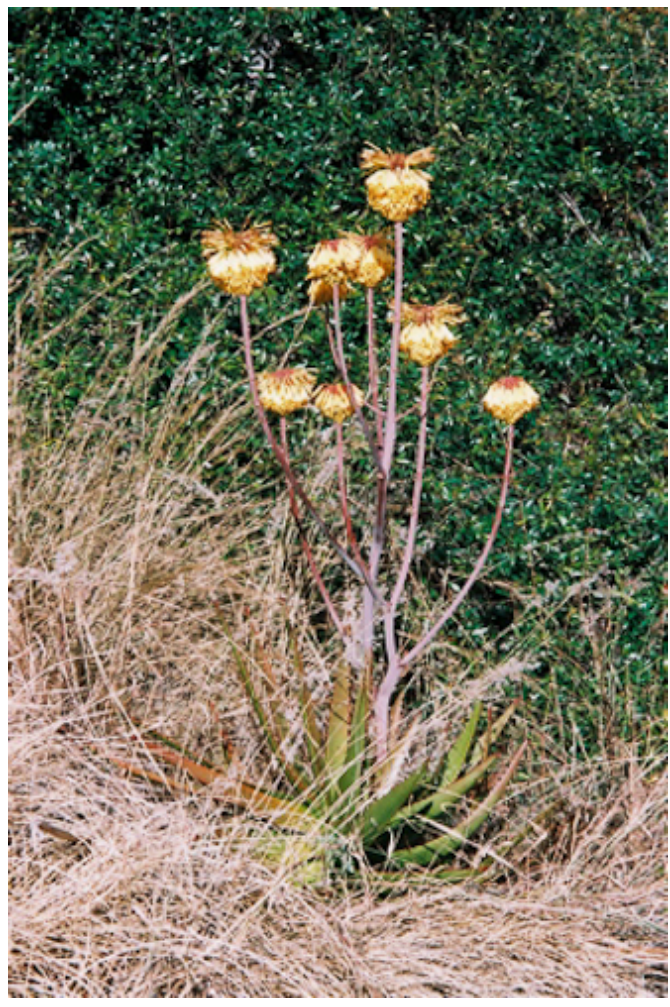

Source : Daniel Peyrusaubes

19 A l'image du kakafotra (Cuculus rochii), ou coucou de Madagascar, qui semble le plus connu des oiseaux, car on y fait immanquablement référence. Le chant de cette espèce endémique serait un signal important de changement de saison. "Quand le premier chant du kakafotra retentit, cela annonce le semis des plants [de riz]» nous dit ce paysan d'ambavala ; c'est donc le passage entre saison peu arrosée et saison humide. Un autre, dans le "pays vieux », précise : «... pour moi le coucou qui chante en octobre c'est deux possibilités : s'il chante au bas de l'arbre, il y aura peu de pluie, s'il chante au sommet, il y en aura beaucoup !» (fig.9). 
Figure 9 - Le kakafotra (Cuculus rochii) chante le retour des pluies

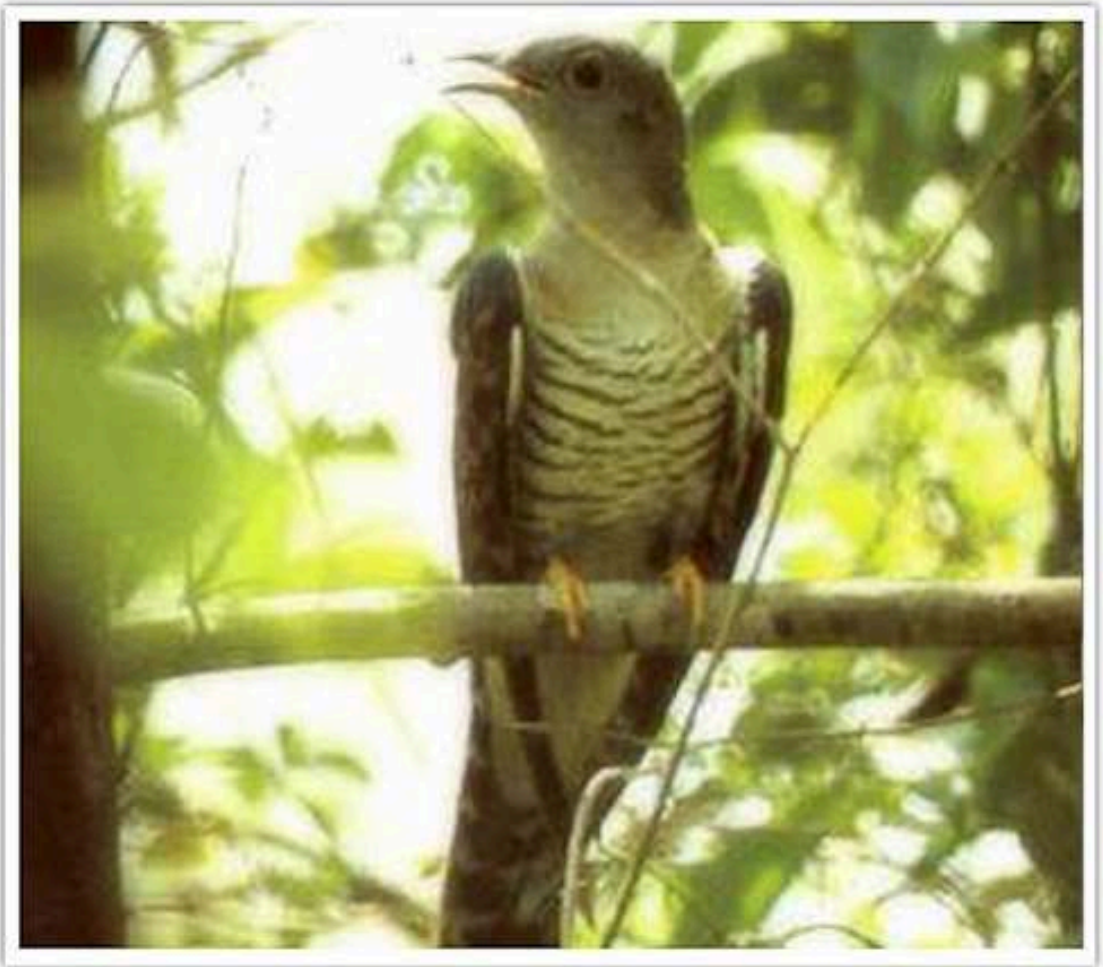

Source : http://www.madagascar-vision.com/

20 A leur tour, les hirondelles, et l'arrivée en masse de libellules (angidina) provenant de l'ouest, annoncent l'imminence des premières pluies de saison chaude. Le comportement et/ou l'apparition de quelques autres animaux et insectes (serpent, scorpion, hannetons, tsibolo $^{8}$ ) participent de ces repères environnementaux auxquels sont sensibles les ruraux merina. Les apiculteurs, quant à eux, font leurs prévisions de changement de saison grâce aux abeilles. Un couple de paysans pionniers rencontrés en " pays neuf » (c'est-à-dire en terres orientales merina) raconte : «Quand mars arrive, en hiver [notons au passage ce repère saisonnier], les abeilles vont à l'ouest, où les fleurs sont ouvertes; on ne peut pas les retenir. Et elles reviennent en octobre. Les premières viennent de passer [l'entretien se déroule en septembre], et l'on a une reine dans notre ruche depuis hier.»

\section{De la culture des champs à une culture « météoroclimatique » orale essentielle... extraits}

Polysémique par excellence, le substantif « culture " nous donne ici l'occasion de l'utiliser par ses deux facettes les plus habituelles. La première concerne l'action de cultiver la terre; la caractéristique agraire du terrain de recherche mobilise, par conséquent, l'acception culturale du mot, d'où l'expression «culture des champs ». La seconde relève du sens intellectuel attribué à « culture »: ensemble des phénomènes et savoirs artistiques, religieux, sociaux... propre à une civilisation, une société, un peuple. L'idée de culture "météoroclimatique ${ }^{9}$ participe de ce champ-là. L'identité agricole des communautés du pays d'Ambohimiadana/Tsiazompaniry est un fait indéniable. En revanche, si quelques indices solides relevés au cours des entretiens effectués auprès 
des habitants donnent à penser à l'existence d'une culture en lien avec le temps qu'il fait, l'esprit de recherche incline à confirmer cette impression ressentie sur le terrain. Aussi, avons-nous exploré d'autres pistes d'ordre culturel susceptibles de mettre au jour une culture météoroclimatique avérée chez cette population de l'Imerina oriental. Comme les chercheurs géographes qui ont travaillé sur les Hautes Terres, en particulier en Imerina, nous avons relevé l'extrême «... importance de la météorologie dans le processus de production et dans la vie quotidienne... » (Rakoto Ramiarantsoa, 1995). La promptitude, la spontanéité, et surtout la qualité des réponses données par les individus témoignent précisément de ce niveau de météorosensibilité. Mais cela ne suffit pas pour pouvoir affirmer l'existence d'une réelle culture en ce domaine ; il faut d'autres témoins, tant dans l'univers langagier que celui de l'écrit. Aussi avons-nous orienté nos recherches dans ces deux directions.

Précisons d'abord ce que nous entendons par «culture météoroclimatique». Ce concept exprime la réalité, face aux multiples états de l'atmosphère, d'un ensemble de faits, tant de comportements et de perceptions individuels et/ou collectifs que de références dans le langage parlé et écrit. Ces faits intéressent aussi bien les phénomènes météorologiques (temps court) que climatiques (temps long), même si ces derniers sont sujets à caution du fait des limites de la mémoire humaine. Cet ensemble de "signes" devient alors un marqueur identitaire du groupe. D'autre part, nous considérons que, par leur mode et niveau de vie et par leurs activités professionnelles, les communautés agraires des pays du Sud pourraient être celles qui, de nos jours, affichent les cultures météoroclimatiques les plus riches et les plus vivantes ${ }^{10}$. Le terrain et les rares références bibliographiques révèlent que les éléments les plus nombreux de cette culture sont les proverbes (ohabolana), dont la richesse traduit une oralité très développée dans la société malgache, ainsi que l'importance des discours (kabary). Très ancienne, "... la maîtrise du verbe fut un véritable moyen de gouvernance... » (Joignerez \& Rajaonarison, 2002), à tel point que parfois les mots suffisaient, comme le suggère l'expression " Ny vava soa sakafo » signifiant « Des paroles bien dites suffisent à apaiser la faim » (ibid.). Les références proverbiales tendent à se faire un peu moins fréquentes aujourd'hui ; les discours, eux, sont toujours aussi présents. Sur le climat, les proverbes évoquent préférentiellement les saisons, soit directement par un trait météorologique particulier, soit indirectement par l'intermédiaire d'un moment de la vie quotidienne ou d'une observation de la nature. Leur compréhension n'est donc pas toujours aisée, leur traduction littérale parfois bien énigmatique. Voici quelques exemples, issus des entretiens et d'un ouvrage spécialisé dans les proverbes ${ }^{11}$, et que nous tentons de déchiffrer :

Lohatoana :

Ny lohataona toy ny voahangy raraka -Le printemps est pareil à une perle tombée à terre.

Le réflexe est de pencher pour la ramasser... il faut donc aussi "ramasser " le printemps, c'est-à-dire en profiter pour préparer les rizières et planter. Cette saison n'est pas « la tête de l'année » pour rien!

Ny kankafotra no famantaran-taona - Le coucou annonce l'année.

Nous retrouvons par cette expression l'importance, au moins dans les cœurs et les esprits, de cet oiseau si souvent cité par les habitants comme le signe avant-coureur du printemps. 
Fahavaratra : extrait d'une chanson interprétée, à la fin des années 1970, par Karison (chanteur d'origine androy) :

Midona ny any ankaratra - Il tonne dans l'Ankaratra

Manako any lavitra any - Et le grondement raisonne au loin.

Fa oram-pahavaratra - C'est une pluie de fahavaratra

No mamonto izany tany - Qui imbibe le sol,

Ny masoko kosa no vonton-dranomaso - Mais mes yeux sont pleins de larmes

Alaelo no miavosa vokatry ny ankaso - Une tristesse m'accable, fruit du malheur.

Fa oram-pahavaratra - Et la pluie d'été,

no mikija sesilany - N'arrête pas de tomber.

Fa ilay volana tomaratra - La lune qui se lève,

Dia indro fa takona any- S'en trouve voilée.

\section{Toutes saisons :}

Tsy ho tapaka angady lohantaona anie hianareo - Que votre bêche ne soit pas abîmée au début de l'année,

$\mathrm{Na}$ ho vaky vilia fararano - Que votre vaisselle ne soit pas cassée en période des dernières eaux,

Tsy ho rovi-damba ririnina koa sombinaina - Que vos vêtements ne soient pas déchirés en hiver,

Na ho tra-pahoriana fahavaratra - Que vous ne soyez pas en deuil pendant la saison des pluies.

massif de l'Ankaratra situé à une cinquantaine de $\mathrm{km}$ à l'ouest du lac de Tsiazompaniry). Les pluies y sont décrites comme copieuses (“... qui imbibe le sol ...”), à l'image des larmes de l'auteur. Quant à la scène de la lune voilée, elle illustre 位

Oram-pararano: rehefa mifanandrify dia latsaka - Les pluies des dernières eaux : quand leurs positions s'accordent, elles tombent. lointaines, seules quelques pluies occasionnelles marquent ce moment au gré des lieux.

Tsy main-tsy lena toy ny andro ririnina - Ni sec ni mouillé, comme un jour d'hiver. concerne surtout la partie orientale des Hautes Terres, là où l'alizé souffle fort et per en un court moment, transformer un ciel bleu azur matinal en un plafond nuageux gris et bas accompagné d'une forte humidité (erika). Le choix de l'adjectif 'mouillé, et non pas 'trempé' par exemple, indique qu'il ne peut s'agir de fortes précipitations, mais plutôt de fines bruines.

Ces paroles ${ }^{12}$, prononcées par un ancien au cours d'une fête rituelle, pourraient être une synthèse simplifiée des relations entre climat et société en Imerina. A chaque saison est associée une idée-maîtresse renseignant sur le fonctionnement de cette société agraire : le printemps et l'angady ${ }^{13}$, car c'est l'époque du labour des rizières, l'automne et la vaisselle, car c'est le moment des grands repas, l'hiver et les vêtements, 
car il faut bien se protéger du froid, l'été et le deuil, car les greniers sont vides de riz et une cérémonie d'enterrement nécessite de nourrir convenablement les invités.

Ces quelques références littéraires ainsi que de l'oralité quotidienne expriment ce potentiel de connaissances des paysans en lien avec le climat. Mélange de sentiments, de vécus et d'observations empiriques de la nature, ce potentiel culturel enfoui au plus profond des âmes paysannes traduit une véritable richesse scientifique. Dès lors, la question est : ce corpus de connaissances vernaculaires est-il en phase avec le savoir scientifique et technique actuel en matière de climatologie de l'Imerina?

\section{La saisonnalité du climatologue, dans la profondeur de l'atmosphère des Hautes Terres}

Selon Tabeaud (2000), le terme saison a deux acceptions: soit il s'agit d'une «... période astronomique comprise entre équinoxe et solstice.», soit c'est une «... période définie dans le cycle annuel par certains états de l'atmosphère, certains types de temps.». Notre choix méthodologique a privilégié l'étude des trois paramètres météorologiques majeurs du climat de l'Imerina (précipitations, températures et vents) ainsi que le décryptage des types de temps caractéristiques des différentes saisons. Seuls les faits essentiels de l'étude analytique sont présentés ici.

A la surface de la Terre, dépressions et anticyclones se partagent continents et océans dans un apparent ordre établi, mais révèlent une "respiration" au gré des saisons. Celle-ci est par exemple bien perceptible dans l'hémisphère austral, avec la dilatation en saison fraîche des trois cellules anticycloniques subtropicales dites de l'île de Pâques, de Sainte Hélène et des Mascareignes. Le résultat en est une ceinture presque continue de pressions élevées induisant une marque saisonnière, singulière, aux espaces concernés. Cette translation, en lien direct avec le déplacement apparent du soleil, s'effectue vers l'équateur en hiver et s'en écarte en été. Ce mouvement atmosphérique annuel de surface s'observe nettement pour l'anticyclone du bassin sudoccidental de l'océan Indien. La position de ce centre d'action barométrique n'est qu'une moyenne (fig.10). 
Figure10 - Champ de pression moyen en Afrique australe en janvier (haut) et juillet (bas)
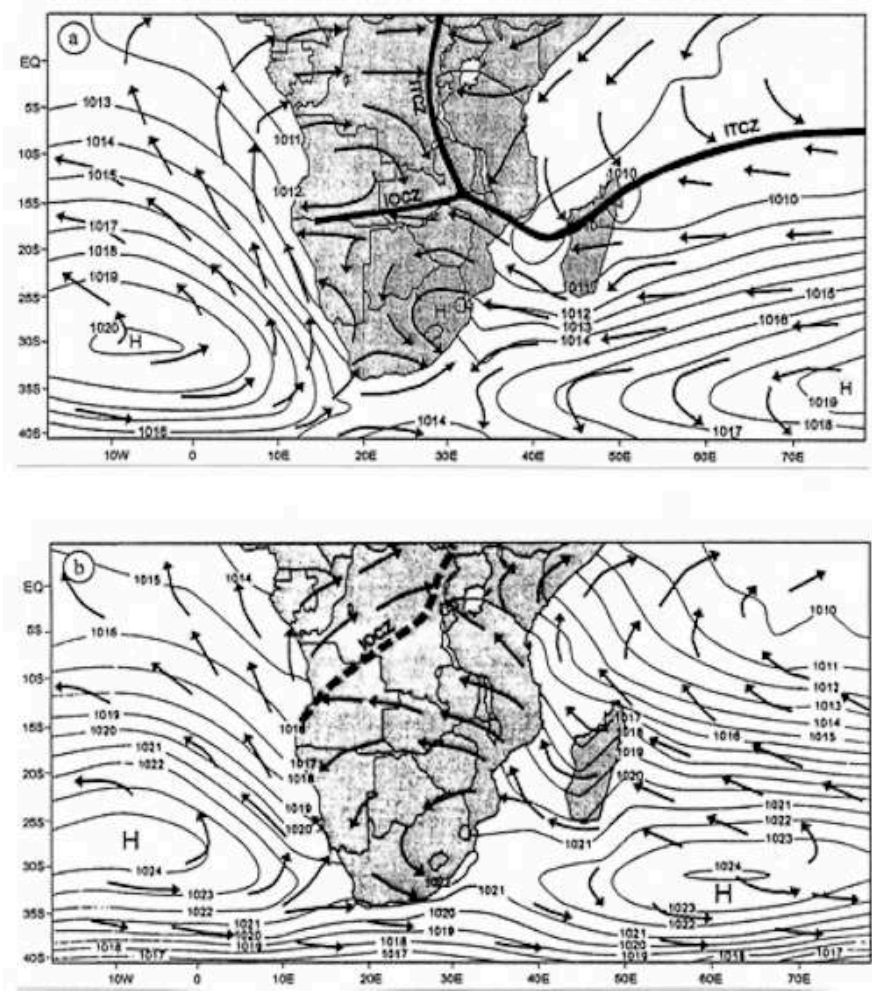

ITCZ est l'acronyme anglais de ZCIT. Les flèches indiquent la direction générale des vents en surface Source : Karoly (1998) des valeurs atteignant jusqu'à $1045 \mathrm{hPa}$ en plein hiver, ou une position centrale bien au-delà à l'est du $70^{\circ}$ est durant la période chaude (la pression maximale peine alors à atteindre $1018 \mathrm{hPa}$ ). Toutefois, ces hautes pressions indiennes « .... sont tributaires du courant de perturbations polaires...» (Donque, 1975) qui s'organise au-delà des quarantièmes « rugissants »; elles sont, en effet, régénérées par l'arrivée d'air froid ou frais méridional post-fronta ${ }^{14}$. Les dépressions méridionales, dirigeant ces systèmes perturbés venus par le sud-ouest, représentent le deuxième centre d'action de surface de cette partie de l'océan Indien austral. Dans le seul cas des talwegs les plus profonds, la marge septentrionale de ces perturbations polaires (généralement l'extrémité des fronts froids) peut atteindre le canal du Mozambique, dont le minimum dépressionnaire constitue le troisième centre d'action régional (estival essentiellement) influençant la climatologie de Madagascar. Enfin, sur son flanc septentrional, l'anticyclone des Mascareignes s'accole à la zone des basses pressions équatoriales qui s'étend très sensiblement vers le canal du Mozambique en été. En cette saison, ce champ dépressionnaire a un rôle capital dans la dynamique climatique de la grande île, plus particulièrement dans les deux-tiers nord. Ce contact se matérialise par une discontinuité atmosphérique majeure, l'équateur météorologique (EM) ex-zone de convergence intertropicale (ZCIT).

S'agissant de l'identification d'un régime pluviométrique, elle se réalise habituellement avec les seules moyennes mensuelles. A défaut d'analyse au pas de temps journalier, voire horaire, nous proposons une étude temporelle des précipitations par décades. $\mathrm{Ce}$ choix s'est imposé pour deux raisons : la première est que cette échelle de temps nous 
paraît compatible avec l'objectif d'un découpage saisonnier, et qu'elle est un bon compromis dans la rythmicité moyenne des types de temps sur les Hautes Terres; la seconde est que cette statistique était disponible dès le début de notre recherche, et nous permettait donc d'engager nos travaux. Ainsi, à partir de l'échantillon statistique des 1296 décades pluviométriques des années 1967 à 2002, une moyenne a été calculée puis mise en graphique. Alors que l'approche mensuelle permet tout au plus de distinguer une saison humide et une saison "sèche", le pas de temps décadaire permet, par sa plus grande finesse, de mieux appréhender le découpage saisonnier. Le fort contraste entre mois très pluvieux et mois peu humides s'affine très nettement (fig. $11)$.

Figure1 1 - Précipitations décadaires moyennes, Antananarivo (1967-2002)

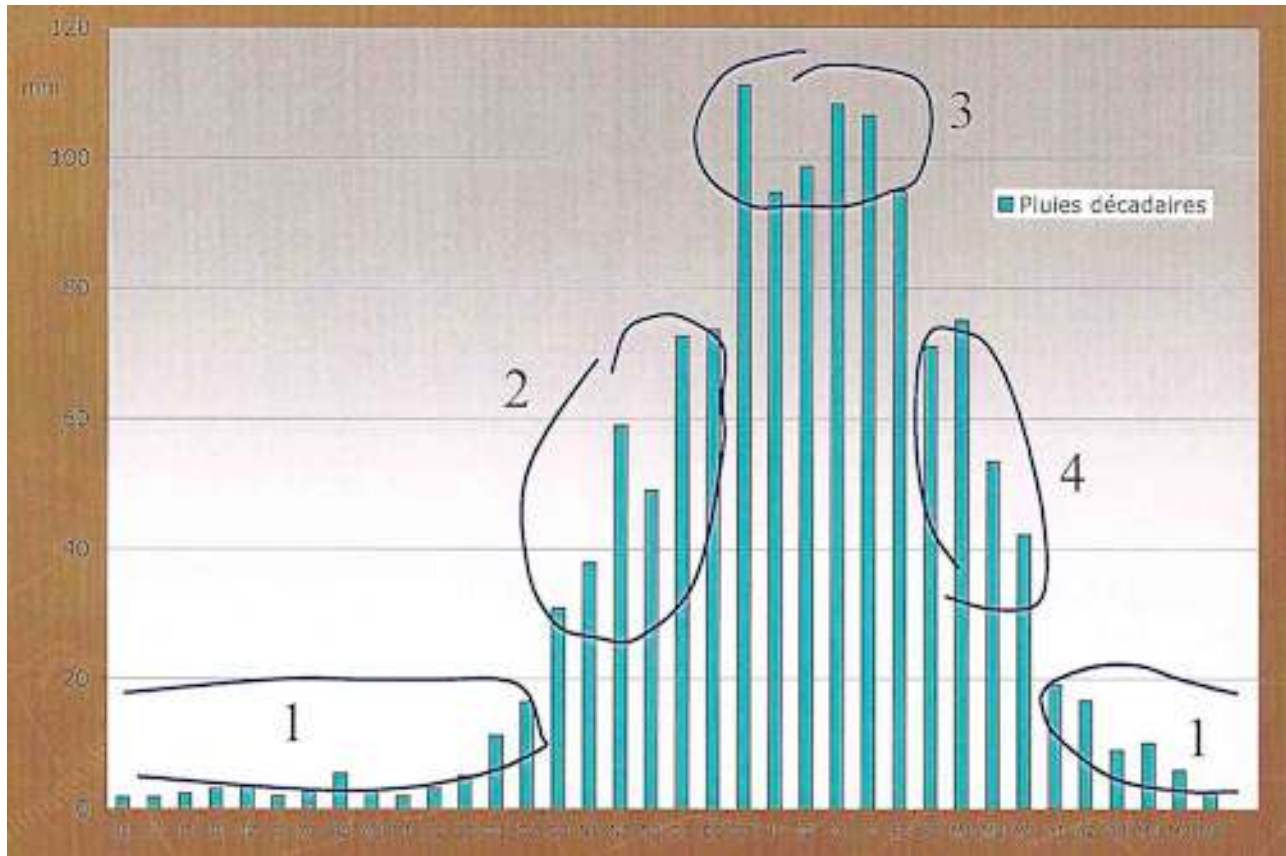

En abscisse, J1 correspond à la première décade de juin, M3 à la troisième décade de mai.

Source : Daniel Peyrusaubes, d'après données Météorologie Nationale (Antananarivo)

L'écart absolu moyen entre décades extrêmes s'établit à 109,5 mm : la 3ème décade de décembre $(111,3 \mathrm{~mm})$, suivie de près par les décades 3 de janvier et 1 de février, enregistre donc en moyenne 62 fois plus de précipitations que les 1ère ou 2ème décades de juin (1,8 $\mathrm{mm}$ chacune). Dans le détail, la distribution pluviométrique montre quelques paliers ou seuils. Ces décrochements statistiques sont suffisamment lisibles. Mais le choix de découpage décadaire peut prêter à discussion car, de manière très empirique, il s'appuie sur une simple lecture directe de l'histogramme. Conscient de cette limite, nous proposons néanmoins la saisonnalité pluviométrique suivante (secteurs numérotés sur le graphique):

- une période peu arrosée court de $\mathrm{A} 1$ (première décade) du mois d'avril à $\mathrm{O} 2$ (deuxième décade) du mois d'octobre ; les pluies moyennes y sont particulièrement médiocres (< $10 \mathrm{~mm}$ ), avec des valeurs un peu plus élevées en début et fin de période, mais toujours inférieures à $20 \mathrm{~mm}$. Rapporté à l'intensité des averses, on peut sans risque évoquer les nombreuses occurrences de bruines et crachins à cette époque, 
- un décrochement statistique en 03 d'octobre préfigure la fin de la saison à faibles précipitations; les décades qui suivent montrent en effet une augmentation régulière et rapide des pluies moyennes mesurées (N3 de novembre se remarque néanmoins par son surprenant retrait). Les niveaux approchent les $75 \mathrm{~mm}$ en D1 et D2 de décembre. Mais un nouveau palier, en D3, nous fait qualifier cette période de transitoire. Elle s'identifie par son régime pluviométrique plus actif, consécutif aux premières incursions de la mousson sur les Hautes Terres centrales malgaches,

- le seuil détecté en $\mathrm{D} 3$ de décembre est particulièrement visible. Par rapport à la décade précédente, la quantité de précipitations reçues augmente de $37,6 \mathrm{~mm}$, soit un tiers en plus ! C'est le cœur de la saison des pluies de la région d'Antananarivo avec une moyenne oscillant autour des $100 \mathrm{~mm}$ par décade. C'est la saison des orages, des pulsations de la mousson, des trombes d'eau. Ce haut niveau pluviométrique se maintient jusqu'en F3 de février où le seuil des $80 \mathrm{~mm}$ n'est plus dépassé. Cette chute statistique, tout aussi nette que la hausse précédente ( $25 \%$ de pluie en moins entre F2 et F3 de février), indique un nouveau changement saisonnier,

- cette nouvelle période de transition que nous limitons à quatre décades (de F3 de février à M3 de mars) est plus courte que son homologue d'octobre/novembre ; les valeurs y sont également un peu supérieures (entre $75 \mathrm{~mm}$ et $42 \mathrm{~mm}$ ). Ce retrait pluviométrique n'est donc pas trop violent, comme si les temps de la saison pluvieuse s'estompaient moins brutalement qu'ils ne semblent s'installer. Cette ultime phase prend fin à la charnière ente M3 de mars $(42 \mathrm{~mm})$ et A1 d'avril $(19,1 \mathrm{~mm}) .$. la ronde saisonnière est ainsi bouclée.

41 Finalement, nous obtenons une partition en quatre périodes de la distribution temporelle des précipitations en Imerina central. Une saison peu pluvieuse et une saison très humide déjà repérées à l'échelle mensuelle, nettement identifiable au niveau décadaire; deux saisons intermédiaires ou de transition, de longueur et de dynamique un peu différentes, mais capitales, surtout pour la première. Et dans les terres rurales environnantes, l'arrivée des premières pluies conséquentes est un moment particulièrement sensible puisque, selon leur date d'occurrence, la nouvelle campagne rizicole peut bien ou mal débuter.

Pour identifier le régime thermique, seule est réalisée une analyse mensuelle, faute de mesures fiables et complètes. La première valeur est la moyenne annuelle, $19^{\circ} \mathrm{C}$ pour notre période 1961/2003, ce qui situe bien Antananarivo dans la zone climatique chaude (ou intratropicale) de la planète, là où le bilan radiatif est excédentaire. L'effet altitudinal s'impose (Antananarivo est à 1276 mètres d'altitude) et, si on applique une décroissance thermique moyenne de $0,5^{\circ} \mathrm{C} / 100$ mètres, on a bien un différentiel de $5^{\circ} \mathrm{C}$ à $6^{\circ} \mathrm{C}$ entre les stations littorales (Toamasina, Maintirano...) et la capitale malgache.

Saison chaude et saison fraîche sont aisément repérables et bien marquées. La première court d'octobre à avril, la seconde est plus courte de mai à septembre. Ainsi, les débuts d'après-midi les plus chauds s'enregistrent aux mois de novembre et décembre $\left(26,2^{\circ} \mathrm{C}\right.$ et $26,1^{\circ} \mathrm{C}$ ), les plus doux au mois de juillet avec une moyenne de $20,2^{\circ} \mathrm{C}$. Concernant les minima moyens, les petits matins de juillet sont les plus frais avec à peine $10,2^{\circ} \mathrm{C}$ sous un ciel clair à dégagé et une atmosphère peu humide (les Merina des campagnes sont alors emmitouflés dans d'épaisses couvertures), alors que l'aube humide et souvent nébuleuse de février est la plus "agréable» de l'année $\left(17^{\circ} \mathrm{C}\right)$. Bref, un régime thermique avec une saison estivale et une saison fraîche. Il n'empêche que la magie d'un climat peut occasionner des surprises, en l'occurrence des situations particulièrement fraîches, voire froides, au point de se poser la question, alors bien 
subjective sur le moment, de la réelle tropicalité du climat de l'Imerina centreoriental $^{15}$ ! Un après-midi très chaud perturbe beaucoup moins la population locale, puisqu'il est tout de même moins inattendu d'avoir chaud que froid sous les tropiques.

Reste à analyser le vent qui est un élément majeur de la climatologie de Madagascar.

La caractéristique la plus remarquable de ce paramètre climatique est sa direction. Rien de révolutionnaire ici puisque l'on connaît, depuis les Grands Navigateurs, le régime de ces vents d'est des latitudes tropicales, les fameux trade winds. Ainsi, Madagascar est, une grande partie de l'année, sous une vaste dorsale de l'anticyclone des Mascareignes. Elle est donc très majoritairement sous l'influence des alizés soufflant d'est à sud-est. Sur les Hautes Terres centrales, aucun mois n'échappe à la prédominance de ce flux, qui en devient même extrêmement prégnant dans la vie quotidienne à certains moments de l'année. En effet, comme pour les deux précédents descripteurs de l'ambiance climatique tananarivienne, nous identifions à nouveau une saisonnalité. Celle-ci s'établit par rapport à la fréquence mensuelle des différentes directions observées quotidiennement. Ces directions, par cadran de $20^{\circ}$, sont visualisées par la mise en graphique de leurs fréquences respectives; les surfaces résultantes sont particulièrement significatives (fig.12).

Figure 12 - Fréquence des directions mensuelles (octobre à septembre) moyennes du vent $\left(^{\circ}\right)$, Antananarivo (1990-2000)
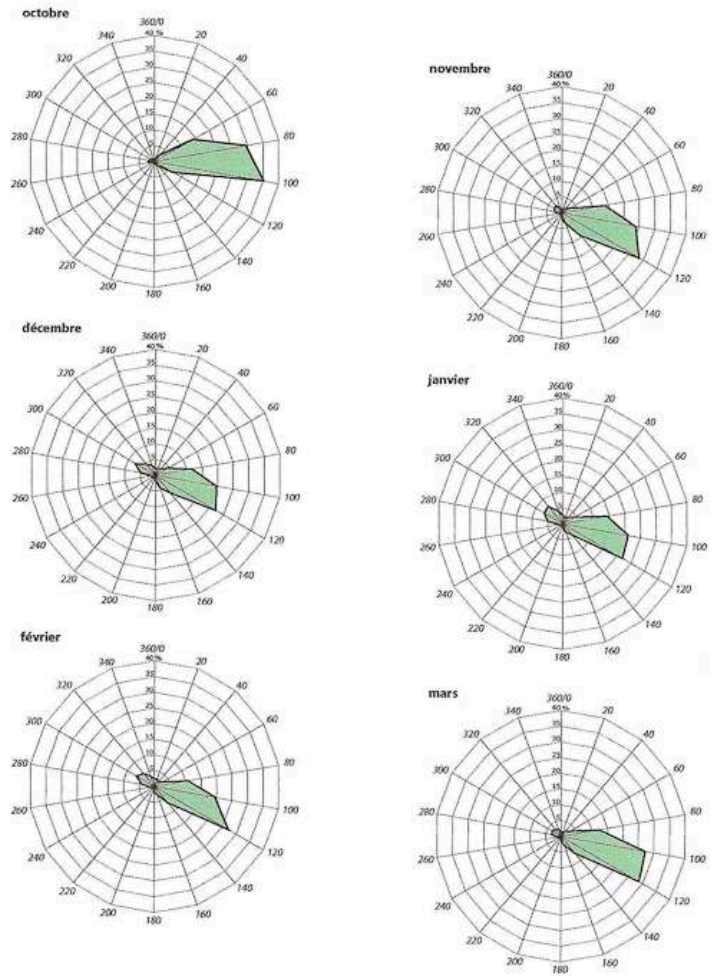

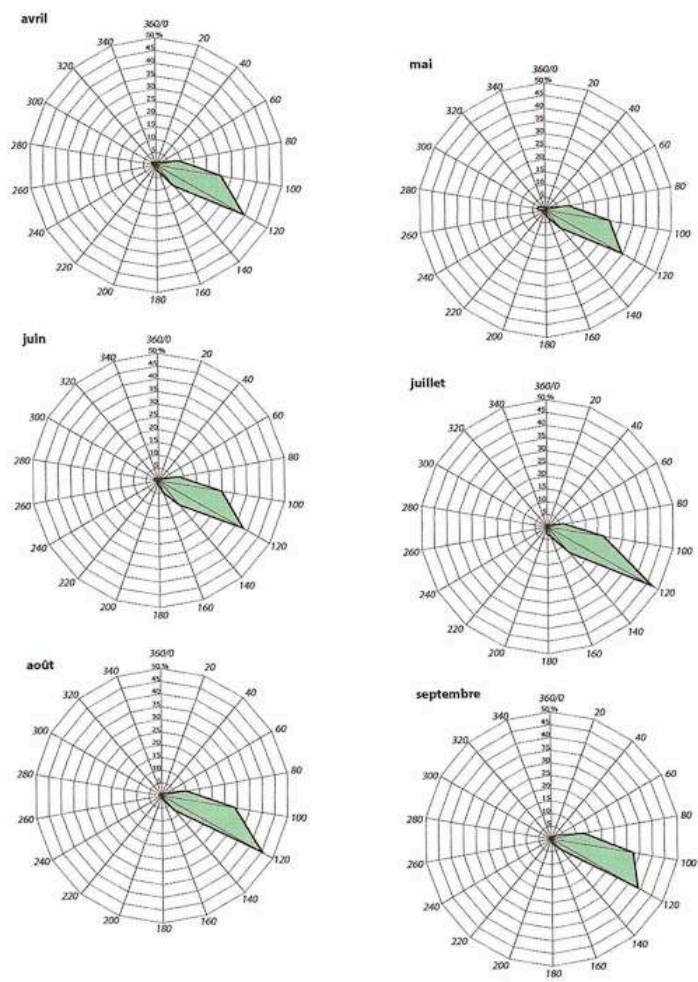

Source : Daniel Peyrusaubes, d'après données Météorologie Nationale (Antananarivo)

La lecture de ces graphiques confirme la présence incontestable de l'alizé tout au long de l'année. Mais, entre les mois de novembre et de mars, on peut observer une « rétraction» visuelle des aires dessinées vers le centre du graphique au profit de l' augmentation consécutive d'une surface quasiment de direction opposée. Puis, d'avril à octobre, s'effectue un mouvement inverse. Telle une respiration, le flux alizéen est donc rythmé, avec une accélération pendant les mois de saison fraîche et peu humide, et un ralentissement durant la période chaude et pluvieuse.

Cette temporalité saisonnière se réalise au profit d'un flux pratiquement opposé en direction, puisque les fréquences en hausse concernent les cadrans $280^{\circ}$ à $340^{\circ}$, soit grosso modo d'ouest à nord-ouest. Dans le détail, cette modification de direction des vents dans la région d'Antananarivo confirme l'existence de deux saisons principales, au sein desquelles les fréquences évoluent lentement, et de deux temps intermédiaires (mars-avril et octobre-novembre) où la structure venteuse se modifie plus radicalement. Ce découpage a été possible par le calcul des fréquences cumulées des deux groupes de directions majeures, et presque opposées : celui de l'alizé entre $80^{\circ}$ et $140^{\circ}$, celui de la mousson entre $280^{\circ}$ et $340^{\circ}$. Derechef, le diptyque alizé/mousson.

Cette image saisonnière est flagrante entre les mois les plus contrastés de janvier et de juillet: respectivement, le vent souffle de l'est à sud-est pour $61 \%$ et $89 \%$ des occurrences, de l'ouest à nord-ouest pour $22,7 \%$ et 1,3\% des cas. L'incursion du flux de mousson, car il s'agit bien du phénomène remarquable de cette étude, a d'autant plus de visibilité (statistique plus que graphique) que la prépondérance de l'alizé est écrasante. Les frémissements de ces vents venus du Canal du Mozambique se repèrent à partir d'octobre où ils approchent $6 \%$ des observations. Le balancement saisonnier interhémisphérique est en train de s'opérer, et les premières bouffées chaudes et humides apportées par ce souffle occidental sur les Hautes Terres centrales malgaches 
traduisent l'avancée vers le sud de l'EM. Pour les trois mois de décembre à février, ce nouveau régime venteux s'établit autour des $20 \%$.

Mars annonce un changement. En effet, l'occurrence des vents alizéens fait une remontée spectaculaire de près de 12 points $(63,6 \%$ à $75,2 \%)$. Le mois d'avril confirme cette tendance avec presque autant de puissance, et ouvre en quelque sorte la période où le flux oriental règne quasiment sans partage (valeurs entre $85 \%$ et $90 \%$ ). Exception: en mai, ce changement atmosphérique de surface se renverse momentanément. Aberration statistique? phénomène climatologique singulier? Seule une étude sur une période plus longue pourrait éventuellement y répondre. Ces mois de fraîcheur et d'assèchement de l'air s'accompagnent donc d'un plein régime d'est à sudest. Cette mécanique est largement commandée par la consolidation des hautes pressions indiennes, dont l'apophyse déployée en Imerina engendre une nouvelle dynamique ambiancielle. Celle-ci s'installe jusqu'en octobre/novembre où, pareillement, un jeu de bascule fréquentiel préfigure le déclenchement d'une nouvelle saison des pluies. Le flux alizéen perd 10 points de fréquence, celui de la mousson en gagne environ 5. Et, presque à l'image du métronome qui donne le tempo, ce changement subtil mais indéfectible dans le régime du vent s'inscrit tant dans la temporalité du tempus que dans le changement du temps qu'il fait, c'est-à-dire de l'ambiance.

50 Mais au-delà d'une étude climatique en surface, notre recherche sur la saisonnalité climatique en Imerina centre-oriental s'est orientée vers une exploration de la dynamique de l'air dans les premiers kilomètres de la troposphère. Nous avons confronté des données pluviométriques de surface à d'autres descripteurs météorologiques mesurés à différents niveaux altitudinaux. Ces mesures sont réalisées au cours des radiosondages effectués à la station ASECNA ${ }^{16}$ localisée sur la plate-forme aéroportuaire d'Antananarivo-Ivato. Les paramètres retenus sont au nombre de quatre : deux valeurs d'instabilité massique (Showalter Index et Precipitable water), la température, l'humidité relative. Ce choix tient à la régularité assez satisfaisante des mesures de ces données et à leur intérêt supposé dans la caractérisation de l'instabilité d'une masse d'air et de son potentiel précipitant. En déplaçant la lecture de la saisonnalité pluviométrique du niveau horizontal (surface) à un niveau vertical, on cherche à savoir si des corrélations entre ces paramètres météorologiques sont susceptibles d'affiner et de confirmer la temporalité déjà identifiée au sol.

51 Nous présentons ici seulement les résultats concernant la température et l'humidité relative mesurées par radiosondage à deux niveaux barométriques standards en météorologie (surface $700 \mathrm{hPa}$ et surface $500 \mathrm{hPa}$ ). Ce choix barique se justifie par le fait que la troposphère est la couche où s'opère la majorité des phénomènes météorologiques producteurs du temps sensible, et en particulier dans les premiers 6000 mètres où se concentre la moitié de la masse atmosphérique.

\section{Dans la couche turbulente}

Les premières centaines de mètres au-dessus de la surface terrestre sont celles où les processus, qui se déroulent dans le fluide atmosphérique, sont les plus nombreux, les plus actifs, les plus puissants, les plus changeants, ceux qui sont les plus influencés par la nature du substratum. Bref, nous sommes dans ce qui est nommé la couche limite planétaire, d'épaisseur variable (500 à 1500 mètres) selon les latitudes, les saisons, les 
conditions du temps, le bilan d'énergie... (Hufty, 2001). S'y déroule, en particulier, la majeure partie des échanges régionaux de chaleur et d'humidité (advections).

Les valeurs calculées à partir des radiosondages quotidiens (période 1990/2004) ne sont qu'indicatives dans la mesure où, selon les mois, les lâchers de ballons ont été irréguliers. Quoi qu'il en soit, l'évolution mensuelle de la température et de l'humidité relative est suffisamment significative pour qu'on accorde du crédit à ces données statistiques (fig.13).

Figure 13 - Température $(\mathrm{T})$ et humidité relative $(\mathrm{H})$ moyennes mensuelles à $700 \mathrm{hPa}$, Antananarivo (1990-2004)

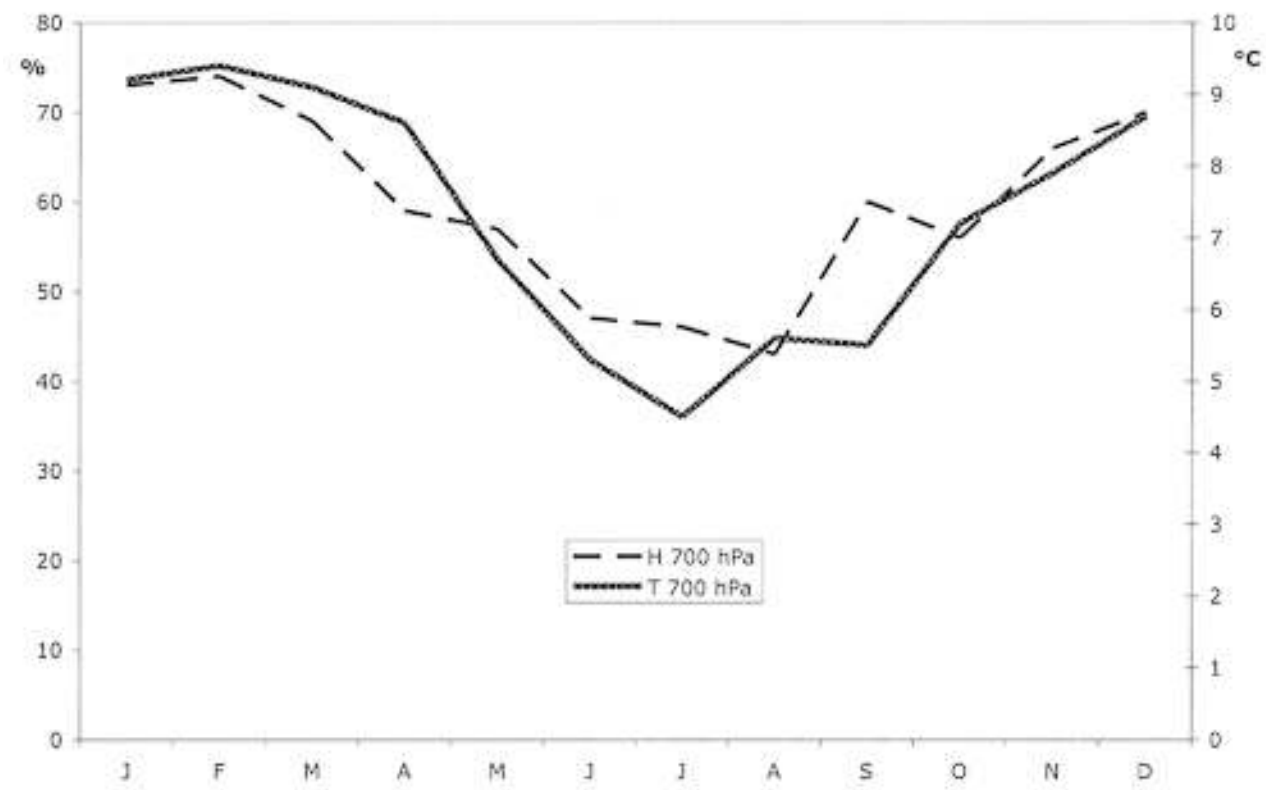

Source : Daniel Peyrusaubes, d'après données de radiosondages

54 L'humidité relative n'est jamais très basse, en moyenne, dans la basse troposphère. La période où l'air est le moins humide se place entre juin et août, où le taux varie de $43 \%$ à $47 \%$. C'est la saison dite "sèche ". De part et d'autre de ce minimum, les taux remontent pour atteindre $69 \%$ à $74 \%$ de décembre à mars. L'opposition entre les deux saisons pluviométriques principales se confirme avec l'hygrométrie moyenne, ce qui n'a rien d'étonnant. En revanche, retrouve-t-on les subtiles périodes de transition? Par une analyse précise, on peut les repérer :

- un pseudo-palier entre avril (59\%) et mai (57\%) traduit une courte partie de l'année pendant laquelle l'assèchement de la masse d'air se ralentit, résultant d'une probable alternance entre ultimes advections humides et premières influences de masses d'air asséchantes,

- un arrêt, entre septembre (60\%) et octobre (56\%), de la franche humidification de l'atmosphère merina déclenchée depuis fin août, signe de séquences sèches contrariant l'évolution massique générale.

Pour sa part, la courbe thermique mensuelle moyenne à ce niveau $700 \mathrm{hPa}$ est d'allure très régulière. Elle présente un maximum de saison chaude en février $\left(9,4^{\circ} \mathrm{C}\right)$, un minimum en juillet $\left(4,5^{\circ} \mathrm{C}\right)$. L'évolution entre ces deux valeurs extrêmes s'effectue linéairement. Une seule exception, le mois de septembre, qui stoppe momentanément 
la remontée des températures. Une hypothèse est que les premières pluies (l'humidité relative augmente de manière concomitante), en limitant l'ensoleillement, ralentissent le réchauffement saisonnier. Octobre, au contraire, fait office de mois nettement plus chaud $\left(+1,7^{\circ} \mathrm{C}\right.$ par rapport à septembre), et ouvre véritablement la saison chaude. La période de transition, en avril/mai, n'apparaît pas.

\section{Aux frontières de l'atmosphère libre}

La surface du géopotentiel $500 \mathrm{hPa}$ se situe à peu près au tiers inférieur de l'épaisseur de la troposphère des Hautes Terres centrales ${ }^{17}$. A cette altitude ( $\approx 5850$ mètres), l'atmosphère ne subit plus de contraintes de la part de la surface terrestre, excepté dans le cas de très hauts reliefs, ce qui n'est pas le cas à Madagascar.

La courbe de l'humidité relative mensuelle se simplifie par rapport à ce qu'elle est à $700 \mathrm{hPa}$ (fig.14). Elle gagne en linéarité - aucun décrochement, signe d'une mécanique atmosphérique apparemment plus régulière - et en amplitude. Cette dernière est particulièrement importante, puisque entre la saison des pluies et la saison moins humide, le taux moyen passe d'environ $60 \%$ à $10 \%$. En moyenne altitude, la saisonnalité pluviométrique est par conséquent clairement identifiable. Les masses d'air envahissant Madagascar de novembre à mars sont franchement beaucoup plus humides et épaisses. L'humidité relative constatée à cette altitude témoigne bien de l'absence de la couche d'inversion de l'alizé, en fait rejetée plus haut. Si bien que les ascendances thermoconvectives ne sont pas contrariées, et peuvent produire des systèmes pluviogènes particulièrement actifs.

Fig.14 - Température $(\mathrm{T})$ et humidité relative $(\mathrm{H})$ moyennes mensuelles à $500 \mathrm{hPa}$, Antananarivo (1990-2004)

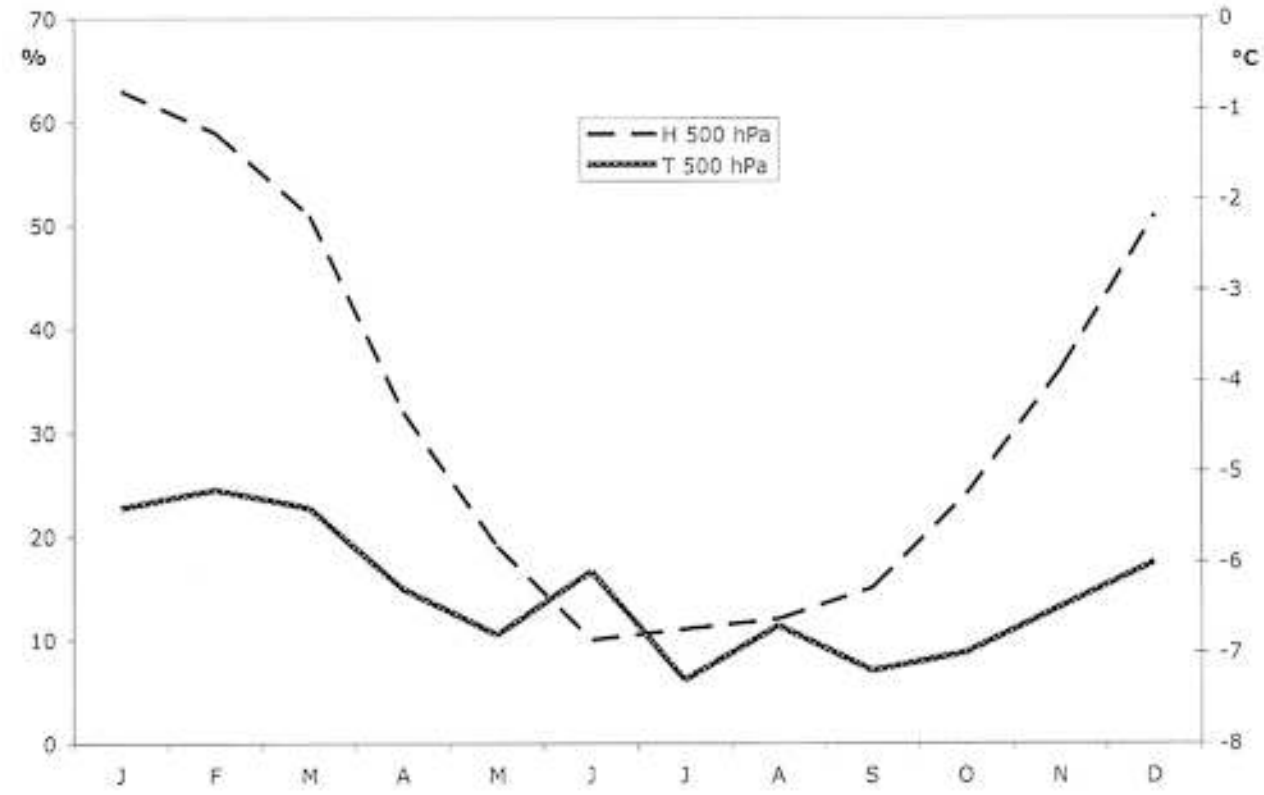

Source : Daniel Peyrusaubes, d'après données de radiosondages

Le phénomène thermique est moins marqué à ce niveau de géopotentiel. Sa distribution moyenne mensuelle dessine une courbure moins accentuée que dans les couches sousjacentes. Son amplitude en est diminuée, puisque le rapport entre extrêmes est de 1,4 (- 
$7,3^{\circ} \mathrm{C}$ en juillet, $-5,2^{\circ} \mathrm{C}$ en février) contre 2,1 à $700 \mathrm{hPa}$. Saisons fraîche et chaude sont donc moins visibles.

A la lecture de cet ensemble d'informations et de connaissances en construction, peuton tenter un bilan du temps des saisons des paysans et du temps des saisons du climatologue?

De manière très empirique, la figure 15 s'y essaie. Ce document représente les ruptures temporelles évoquées dans le discours des paysans et repérées dans l'étude des paramètres météorologiques.

Figure15 - Calendriers climatiques, paysan et analytique, croisent leur regard

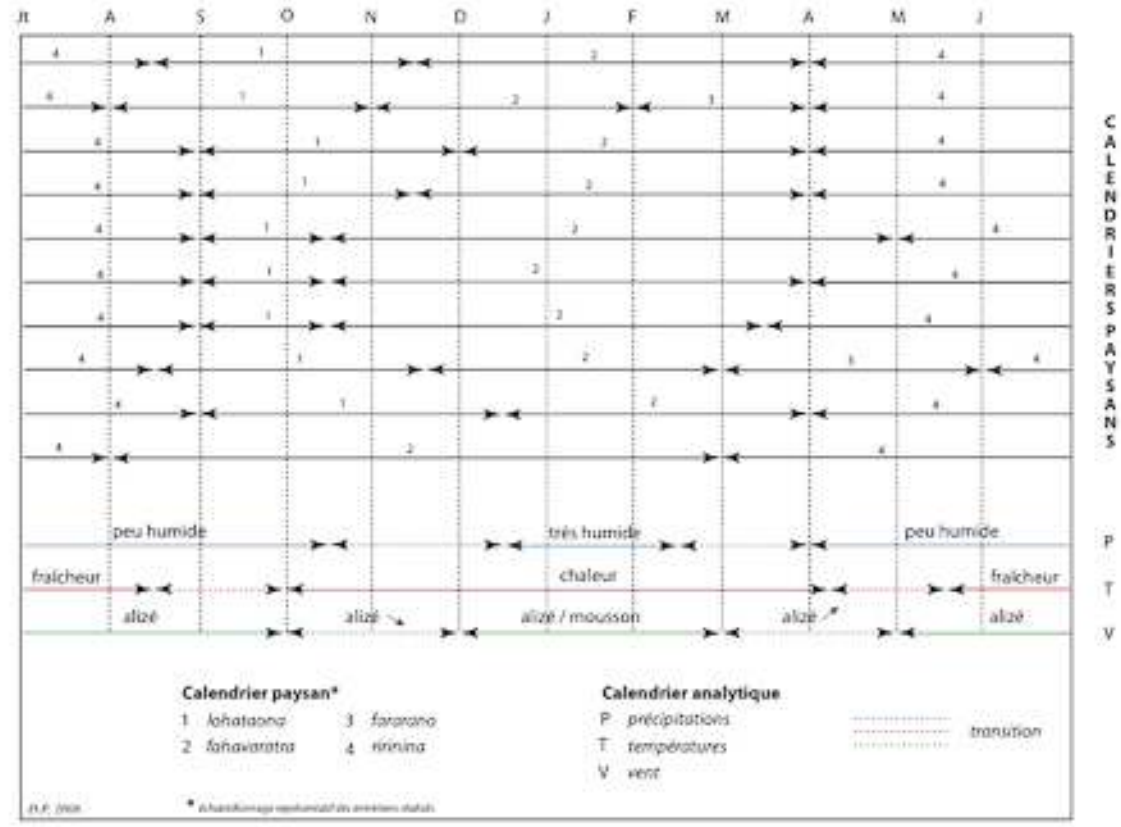

Conception et réalisation : Daniel Peyrusaubes

Le calendrier analytique est une proposition de découpage saisonnier annuel basé sur les trois paramètres essentiels de la région étudiée: les précipitations, la température, le vent. Sa principale limite relève de l'hétérogénéité temporelle de l'analyse de ces trois paramètres: décadaire pour les pluies, mensuelle pour les températures et le vent. De même, les périodes des séries statistiques sont inégales: 1967/2002 pour les précipitations, 1961/2003 pour les températures et 1990/2000 pour le vent.

Quant au découpage paysan, il illustre les principales options citées au cours des entretiens. Quand il s'agit de découper l'année en saisons, les réponses se recoupent à quelques nuances près. Le début septembre marque bien un moment important dans l'ordonnancement saisonnier. Il en est de même pour fin mars/début avril, signal de l'établissement prochain de ririnina. Fahavaratra est ensuite perçu plus ou moins long, de 3 mois à presque 6 mois ! Lohataona, presque toujours cité, ne dure jamais plus de 2 à 3 mois, entre septembre et novembre. L'automne est quasiment absent de ce découpage, et se place entre février et mai quand on l'évoque.

Même si aucune synchronie parfaite ne ressort des divers moments exprimés par ces deux lectures (paysan/scientifique), il y a une correspondance proche entre les 
périodes thermiques que nous qualifions de transition - en septembre et en avril - et le basculement hiver/printemps, tout comme la fin de l'été, bien perçus par les populations. Cela est un peu moins net avec notre découpage saisonnier pluviométrique, le principal moment de non convergence des calendriers se plaçant à la charnière entre saison peu arrosée et saison pluvieuse. C'est manifestement la partie de l'année climatique qui pose le plus de problèmes dans son analyse/perception. Mais n'est-elle pas essentielle pour les paysans? N'est-elle pas le début d'une nouvelle campagne rizicole si essentielle pour l'alimentation future de chacun? D'où une sensibilité plus prononcée de la part des locaux pour ce moment de l'année, et, par conséquent, la probabilité d'une plus grande diversité dans les perceptions individuelles. Quant au vent, l'analyse saisonnière que nous en faisons est en cohérence avec la phase de fin de fahavaratra (période de transition où l'alizé reprend de la vigueur), mais un décalage d'au moins un mois s'inscrit durant le passage saison " sèche "/saison des pluies. Rappelons que ce découpage statistique ne concerne qu'une courte période de onze années (1990-2000), ce qui en limite forcément la pertinence.

Pour conclure, il nous paraît important de rappeler que les études à l'interface de deux domaines différents, des sciences fondamentales comme des sciences humaines, relèvent d'un pari difficile. Notre recherche participe de l'ambivalence, entre physique et sociétal, qui caractérise les géoclimatologues français (Vigneau, 2006). Ce type de travail aboutit à des résultats dont la pertinence n'est pas toujours immédiatement lisible. Les deux lectures du climat montrent des convergences à bien des égards, et une complémentarité évidente. Savoirs vernaculaires et savoirs savants se nourrissent mutuellement, à la condition expresse que les premiers soient identifiés et qu'ils soient diffusés ! Si les traits généraux (pluies, températures, vents...) de la climatologie locale et régionale se confondent peu ou prou entre les deux sources, la richesse des savoirs et $\mathrm{du}$ vécu des paysans merina apportent une finesse de la connaissance de l'environnement. Celle-ci est évidente au début de la saison des pluies, et montre un certain décalage entre statistique et appréhension paysanne. De même, la saison automnale passe presque inaperçue chez ces populations, et la plus grande fraîcheur des contrées orientales merina est bien confirmée par les témoignages. Par ailleurs, l'existence d'une météoroculture régionale encore vivante (proverbes, observations de la faune, de la flore, du ciel...) devrait attirer l'attention des gouvernants, dans un contexte où la vulnérabilité des hommes face à des changements écologiques en cours semble s'accroître. Les nouvelles gouvernances d'adaptation à inventer devront se nourrir de ses savoirs et savoir-faire.

Ce résultat d'ensemble est d'autant plus remarquable que les populations rencontrées n'ont pas de culture du chiffre, de la mesure exacte. Cette convergence/ complémentarité d'appréciation et d'interprétation des "choses du temps » devient dès lors particulièrement motivante pour une exploration approfondie. Finalement, la lecture paysanne du climat en terres malgaches, et hypothétiquement en toutes terres rurales de pays en développement, ne mérite-t-elle pas d'être qualifiée de scientifique au même titre que celle du climatologue ? Sur une planète en plein changement global, il nous semble inconcevable de traiter des questions environnementales sans prendre en compte la manière dont les individus les perçoivent, et ce qu'ils en connaissent. Donner un éclairage sur les savoirs et savoir-faire locaux en matière d'environnement, ici le climat, nous semble une nécessité. Bien de ces savoirs traditionnels, qui révèlent en coulisses des stratégies adaptatives singulières (dans le contexte du changement climatique par exemple), trouvent aujourd'hui une légitimité, donc un intérêt 
scientifique. Dans une autre aire géographique, le cas des peuples nordiques est, à cet égard, particulièrement passionnant. Néanmoins, nous gardons à l'esprit que le champ de la mémoire humaine, qui a été évidemment mobilisé dans cette étude sur le climat et les saisons, limite la portée des propos recueillis. Comme l'illustre fort bien le proverbe mossi suivant: "Quand la mémoire s'en va ramasser du bois mort, elle rapporte le fagot qui lui plait.» (Soudière, 1999). Ce bémol ne remet pas en cause notre posture générale, et le débat reste ouvert

\section{BIBLIOGRAPHIE}

Banc-Pamard C., 1986. Dialoguer avec le paysage ou comment l'espace écologique est vu et pratiqué par les communautés rurales des hautes terres malgaches. in : Chatelin Y, Riou G (Ed.), Milieux et paysages. Paris : Masson, p. 17- 34

Boko M., Bokonou-Ganta E., Pérard J., 1990. Contraintes climatiques et croyances en Afrique tropicale : essai d'ethnoclimatologie. Publication de l'AIC, vol.3, p. 163-171.

Donque G., 1975. Contribution géographique à l'étude du climat de Madagascar. Tananarive, 478 p.

Hébert J.-C., 1968. Les calendriers saisonniers à Madagascar. Bulletin de Madagascar, 260, p. 42-85

Houlder J.-A., 1960. Ohabolona ou proverbes malgaches. Antananarivo : Sibree/D.D/F.R.G.S, 216 p.

Hufty A., 2001. Introduction à la climatologie. Bruxelles : De Boeck Université, 542 p.

Joignerez A., Rajaonarison E., 2002. Voyage en terre malgache, le cœur de l'Imerina. Antananarivo : Aquaterre/Phyto-Logic, $113 \mathrm{p}$.

Karoly D., Vincent D., 1998. Meteorology of the southern hemisphere. American Meteorological Society, vol.27, $49 \mathrm{p}$.

Katz E., Lammel A., Goloubinoff M., 2002. Entre ciel et terre, climat et sociétés. Paris : IRD, 509 p.

Lamarre D., Pagney P., 1999. Climats et sociétés. Paris : Colin, 272 p.

Peyrusaubes D., 2009. Gradient, savoirs et mémoire : le changement observé et perçu dans la climatologie de l'Imerina centre-oriental (Madagascar), in Tabeaud M. (Ed.), Le changement en environnement. Paris : Publications de la Sorbonne, p. 55-66

Peyrusaubes D., 2007. Climatologists and merina farmers read the sky in Madagascar. Crossed glances. XVth International Conference of the SHE "Human Ecology : Local populations and Diversity in a Changing World", Rio de Janeiro 4-7 octobre 2007 http://iri.columbia.edu/taddei/XVSHE/ SHE2007.htm

Rakoto Ramiarantsoa H., 1995. Chair de la terre, oeil de l'eau... : paysanneries et recompositions de campagnes en Imerina (Madagascar). Paris : Orstom. 370 p.

Rakoto Ramiarantsoa H., Peyrusaubes D., 2007. Nuages, vents et pluies : scruter le " visage du firmament " en Imerina. Cahiers d'Outre Mer, 240, p. 319-340

Soudiere (de La) M., 1990. Revisiter la météo. Etudes Rurales, 118/119, p. 9-29. 
Vigneau J.-P., 2006. Eclipse et retour du sociétal en climatologie. Actes des « Journées de climatologie » de la Commission Climat et Société du CNFG, Nice 23-25 mars 2006, p. 25-34.

\section{NOTES}

1. Expression utilisée par certains climatologues français à partir des années 1970. Quatre décennies plus tard, nous la trouvons toujours aussi belle et moderne.

2. Peyrusaubes D, 2007. "Ma part de nuage»: Climat et société en Imerina centre-oriental (Madagascar). Lille : ANRT, 369 p. Ce travail est le fruit d'une thèse réalisée sous la codirection d'Hervé Rakoto Ramiarantsoa et de Jean-Pierre Vigneau, soutenue en janvier 2006 à l'Université de Poitiers

3. $50 \%$ des terres malgaches se situent à plus de $500 \mathrm{~m}$ d'altitude, $20 \%$ dépassent les 1000 mètres (Donque, 1975).

4. Il s'agit de cultures sur rizières, en dehors du cycle cultural du riz, donc en saison peu humide et fraîche; il peut s'agir de pomme de terre, de taro, de brèdes...

5. Ces enquêtes, réalisées à vélo en compagnie de notre collaborateur-interprète, se sont déroulées en trois périodes de quinze jours (janvier 2002, septembre 2002, juin 2003). Une quarantaine d'entretiens ont été effectués auprès de ruraux d'âges différents (30 à 85 ans) et de conditions sociales variées (paysans, fonctionnaires, commerçants, astrologue...). En cohérence avec la recherche d'un gradient climatique, ces entretiens ont eu lieu à l'ouest et à l'est du lac de Tsiazompaniry.

6. Noté dès nos premiers entretiens en 2002, et confirmé par la suite, nous sommes le premier à dialoguer avec cette population de l'Imerina sur le thème du climat.

7. Cette partition climatique basique, une vieille expression merina l'évoque en faisant référence aux premières pluies qui séparent l'année: ranonorana mampisara-taona, litt. «pluies qui divisent l'année» (Hébert, 1968). Se déclenchant en lohataona, ces pluies firent l'objet d'une étude statistique par le Père Colin, sur 28 années d'observations à Antananarivo (1ère moitié du XXe siècle): elles eurent lieu 25 fois en octobre, 3 fois en novembre.

8. Variété de chenille se trouvant dans les bas-fonds, exclusivement en saison fraîche; un autochtone d'Antakafatra ne nous affirme-t-il pas: «quand il y en a beaucoup [de tsibolo], c'est que ririnina est proche»!

9. Cet adjectif est construit à partir de l'expression en grec ancien 'ta meteôra', « les choses qui sont en haut, ou élevées », et qui est la racine du substantif contemporain 'météorologie'. Cette construction est donc préférable à 'météoclimatique'.

10. Voir de très intéressants exemples dans Katz et alii (2002).

11. Houlder, 1960

12. Témoignage recueilli sur le terrain.

13. L'angady est la bêche utilisée lors de la préparation des rizières.

14. Ces régénérations sont paradoxalement plus puissantes en été car, durant la saison hivernale, Madagascar est pour ainsi dire protégée par la barrière de hautes pressions reconstituées de l'Afrique du Sud aux îles des Mascareignes (Donque, 1975).

15. Nous faisons allusion ici à un épisode de terrain particulièrement rude, à l'est du lac de Tsiazompaniry, en situation d'alizé humide très actif de fin de saison fraîche occasionnant des averses de pluies fines particulièrement glaciales.

16. $A S E C N A=$ Agence pour la Sécurité de la Navigation Aérienne en Afrique et à Madagascar.

17. L'altitude de la tropopause s'établit aux environs de $17 \mathrm{~km}$, à une température de $-74^{\circ} \mathrm{C}$ (Donque, 1975). 


\section{RÉSUMÉS}

Décrypter la climatologie d'un espace géographique consiste habituellement en l'interprétation de recherches à partir de corpus de statistiques et d'observations instrumentales. Mais une autre source de connaissances est celle des savoirs des populations locales. Cette posture est appliquée à la région de l'Imerina, sur les Hautes Terres de Madagascar. Ainsi, une double approche climatologique est expérimentée : l'une, à partir des connaissances vernaculaires paysannes, l'autre, via une classique étude statistique. La confrontation de ces deux lectures montre une intéressante complémentarité entre les sources. Et, dans un contexte de changement climatique et de développement durable, être à l'écoute des savoirs et savoir-faire locaux relève d'une posture scientifique plus moderne que jamais.

To decipher the climatology of a geographical area usually consists in interpreting data collected from a corpus of statistics and instrumental observations. However, another source of data is based on the knowledge of the local populations. This posture is applied to the region of Imerina, on the highlands of Madagascar. So, a double climatological approach is experimented: on one hand, based on the peasant vernacular knowledge, on the other hand, via a classic statistical study. Confronting these two analysis shows how different sources of data can be complementary. Furthermore, paying attention to local knowledge and know-how in a context of climate change and sustainable developement is a way to apply a more modern than ever scientific posture.

\section{INDEX}

Keywords : calendar, climatology, local knowledge, Madagascar, season

Mots-clés : calendrier, climatologie, Madagascar, saison, savoir local

\section{AUTEUR}

\section{DANIEL PEYRUSAUBES}

Daniel Peyrusaubes est Maître de Conférences, codirecteur du Département de géographie à l'Université de Poitiers et membre de ICoTEM EA2252. Il a publié récemment : Peyrusaubes D. 2009. Gradient, savoirs et mémoire : le changement observé et perçu dans la climatologie de l'Imerina centre-oriental (Madagascar), in Tabeaud M. (Ed.), Le changement en environnement. Paris : Publications de la Sorbonne, p. 55-66 daniel.peyrusaubes@univ-poitiers.fr 